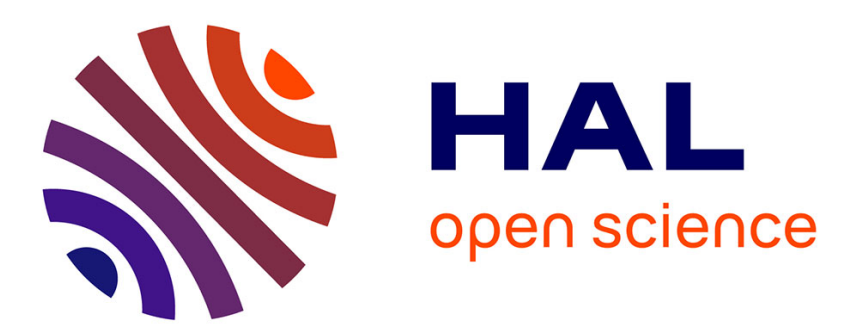

\title{
Individual muscle contributions to ground reaction and to joint contact, ligament and bone forces during normal gait
}

Florent Moissenet, Laurence Cheze, Raphaël Dumas

\section{- To cite this version:}

Florent Moissenet, Laurence Cheze, Raphaël Dumas. Individual muscle contributions to ground reaction and to joint contact, ligament and bone forces during normal gait. Multibody System Dynamics, 2017, 40 (2), pp.193-211. 10.1007/s11044-017-9564-9 . hal-01576285

\section{HAL Id: hal-01576285 \\ https://hal.science/hal-01576285}

Submitted on 22 Aug 2017

HAL is a multi-disciplinary open access archive for the deposit and dissemination of scientific research documents, whether they are published or not. The documents may come from teaching and research institutions in France or abroad, or from public or private research centers.
L'archive ouverte pluridisciplinaire HAL, est destinée au dépôt et à la diffusion de documents scientifiques de niveau recherche, publiés ou non, émanant des établissements d'enseignement et de recherche français ou étrangers, des laboratoires publics ou privés. 


\title{
Individual muscle contributions to ground reaction and to joint contact, ligament and bone forces during normal gait
}

\author{
F. Moissenet ${ }^{1}$, L. Chèze ${ }^{2}$, R. Dumas ${ }^{2}$ \\ ${ }^{1}$ Centre National de Rééducation Fonctionnelle et de Réadaptation - Rehazenter, \\ Laboratoire d'Analyse du Mouvement et de la Posture, Luxembourg \\ 2 Univ Lyon, Université Claude Bernard Lyon 1, IFSTTAR, UMR_T9406, LBMC, F69622, Lyon, \\ France
}

\section{Corresponding author:}

Florent Moissenet

Centre National de Rééducation Fonctionnelle et de Réadaptation - Rehazenter, Laboratoire d'Analyse du Mouvement et de la Posture, 1 rue André Vésale, L-2674, Luxembourg, Luxembourg

+352269894310

florent.moissenet@mailoo.org

\begin{abstract}
Recent developments in musculoskeletal modelling have enabled numerous studies to explore how individual muscles contribute to progression, support and mechanical loading during gait. However, the literature still lacks data on the contributions of musculo-tendon forces to several structures, making it difficult to determine the primary contributors. The aim of the present study was thus to provide a comprehensive investigation of individual muscle contributions to ground reaction (i.e. 3D ground reaction force and moment), and to joint contact, ligament and bone (i.e. compression-traction of bony segments) forces during normal gait. We used a 3D lower limb musculoskeletal model coupled with a static optimisation method using a pseudo-inverse, which indeed yielded data on individual muscle contributions currently missing from the literature. We report the individual muscle contributions to 1) 3D ground reaction force and moment, 2) hip, tibiofemoral, patellofemoral, and ankle joint contact forces, 3) tibiofemoral and ankle ligament forces and 4) femur, patella and tibia bone forces. In line with the recent literature, the primary contributors are the vastii, gluteus medius, soleus, rectus femoris, gemellus, quadratus femoris, gluteus maximus and adductors. While the current observations are made on a generic model, the present method offers a comprehension tool that can shed light on the underlying mechanisms governing the musculoskeletal system.
\end{abstract}

Key terms: Individual muscle contribution, Pseudo-inverse method, Musculoskeletal modelling, Static optimisation, Gait 


\section{Introduction}

To better understand the mechanisms underlying gait, it is important to investigate how individual muscles contribute to progression, support, and mechanical loading [1, 2]. This could provide insights into pathologic gait patterns [3], as well as the progression of joint disorders such as osteoarthritis [1, 4]. Several studies have analysed how musculo-tendon forces contribute to ground reaction force [2, 3, 5-7], acceleration of the centre of mass [8-11], whole-body angular momentum [12], angular accelerations of joints [13], linear and angular accelerations of segments [6], and joint moment at the ankle, knee and hip [2, 4, 14, 15]. Studies have also analysed how musculo-tendon forces contribute to 3D hip joint contact force $[2,16]$, the vertical component of 3D tibiofemoral joint contact force [2, 4, 14, 17-19], the vertical component of 3D ankle joint contact force [2] and femur force [20]. However, to the authors' knowledge, no study has to date analysed how individual muscles contribute to 3D patellofemoral contact force, patella and tibia forces, and only one study investigated how some of the musculotendon forces (i.e. soleus and gastrocnemii) contribute to ligament forces (i.e. anterior cruciate ligament) during single-leg standing [21].

Most of the existing studies are based on a forward dynamics process with induced acceleration analysis [2, 5, 6, 16] or perturbation analysis [3, 9, 13]. However, Lin et al. [7] recently proposed an alternative method based on inverse dynamics and a static optimisation method using a pseudo-inverse. This method is attractive, not entailing high computational costs and enabling investigation of how musculo-tendon forces contribute to ground reaction (i.e. 3D ground reaction force and moment), as well as to any joint contact, ligament and bone forces in the inverse dynamics formulation.

Here, individual muscle contributions missing from the literature were computed. For that, the pseudo-inverse method [7] was used on a 3D lower limb musculoskeletal model which particularity is to simultaneously estimate musculotendon forces and joint contact, ligament and bone (i.e. compression-traction of bony segments) forces [22, 23]. Our aim was to provide a comprehensive investigation of how individual muscles contribute both to ground reaction and to joint contact, ligament and bone forces during normal gait.

\section{Materials and methods}

\section{Musculoskeletal model}

A previously described [22, 23] 3D lower limb musculoskeletal model consisting of 5 segments (i.e. pelvis, thigh, patella, shank and foot) and 5 degrees of freedom was used to perform this study. Hip, tibiofemoral, patellofemoral and ankle joint kinematic models were all based on anatomical considerations (see Supplementary material). Muscular lever arms were computed using a muscular geometry derived from that proposed by Klein Horsman et al. [24] and adjusted by van Arkel et al. [25]. This geometry consisted of 129 muscular lines of action representing 38 muscles, divided into 55 units with up to 6 bundles. Both hip and knee contact forces estimated with this model were validated against instrumented prosthesis measurements $[22,23]$. The muscular redundancy problem was solved 
at the musculo-tendon forces level, without the use of a muscle model. The related optimisation procedure is described below.

\section{Simultaneous estimation of musculo-tendon, joint contact, ligament and bone forces}

The dynamics equation for the lower limb was written to introduce the musculo-tendon forces and Lagrange multipliers [26, 27]:

$\mathbf{G} \ddot{\mathbf{Q}}+\mathbf{K}^{T} \boldsymbol{\lambda}=\mathbf{R}+\mathbf{P}+\mathbf{L f}$

where $\mathbf{G}$ is the matrix of generalised masses, $\ddot{\mathbf{Q}}$ is the vector of generalised accelerations, $\mathbf{K}^{T}$ is the Jacobian matrix of both kinematic and rigid body constraints, $\boldsymbol{\lambda}$ is the vector of Lagrange multipliers, $\mathbf{R}$ is the vector of generalised ground reaction (i.e. including the $3 \mathrm{D}$ force $\mathbf{F}_{0}^{\mathbf{R}}$ and moment $\mathbf{M}_{0}^{\mathbf{R}}$ at the centre of pressure (CoP)), $\mathbf{P}$ is the vector of generalised weights, $\mathbf{L}$ is the matrix of generalised muscular lever arms and $\mathbf{f}$ is the vector of musculo-tendon forces. Note that the model is made of 5 segments but the dynamics equation is written excluding the pelvis (which is only required for the definition of the hip joint constraints and some muscle origins).

At this level, a parameter reduction [28] can be used to cancel all the Lagrange multipliers from equation 1, defining the musculo-tendon forces $\mathbf{f}$ as the only unknowns. However, one part of these Lagrange multipliers, corresponding straightforwardly to the joint contact, ligament and bone forces (i.e. $\lambda_{1}$ ) [22] can be kept as unknowns using a partial parameter reduction [22]:

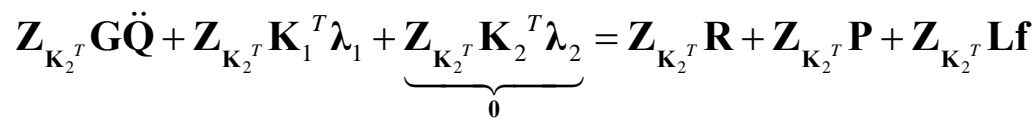

$$
\begin{aligned}
& \Leftrightarrow \mathbf{Z}_{\mathbf{K}_{2}{ }^{T}}\left[\begin{array}{ll}
\mathbf{L} & \mathbf{K}_{1}^{T}
\end{array}\right]\left[\begin{array}{c}
\mathbf{f} \\
\boldsymbol{\lambda}_{1}
\end{array}\right]=\mathbf{Z}_{\mathbf{K}_{2}{ }^{T}}(\mathbf{G} \mathbf{Q}-\mathbf{P}-\mathbf{R})
\end{aligned}
$$

where $\lambda_{1}$ is the vector of the Lagrange multipliers standing for the joint contact, ligament and bone forces of interest ( $\boldsymbol{\lambda}_{2}$ is the vector for all the others), $\mathbf{K}_{1}$ and $\mathbf{K}_{2}$ the associated Jacobian matrices and $\mathbf{Z}_{\mathbf{K}_{2}{ }^{T}}$ is the orthogonal basis of the null space of $\mathbf{K}_{2}{ }^{T}$. The Lagrange multipliers $\boldsymbol{\lambda}_{1}$ were the same as in [22] (i.e. contact force of ankle, tibiofemoral, patellofemoral and hip joints, ligament force of ankle, tibiofemoral and patellofemoral joints, and bone forces).

The unknowns $\left[\begin{array}{ll}\mathbf{f} & \lambda_{1}\end{array}\right]^{T}$, corresponding respectively to the musculo-tendon forces and the selected joint contact, ligament and bone forces, were then introduced in a one-step optimisation procedure in order to solve the muscular redundancy problem. A typical static optimisation procedure was used and defined as follows $[26,27,29]$ : 


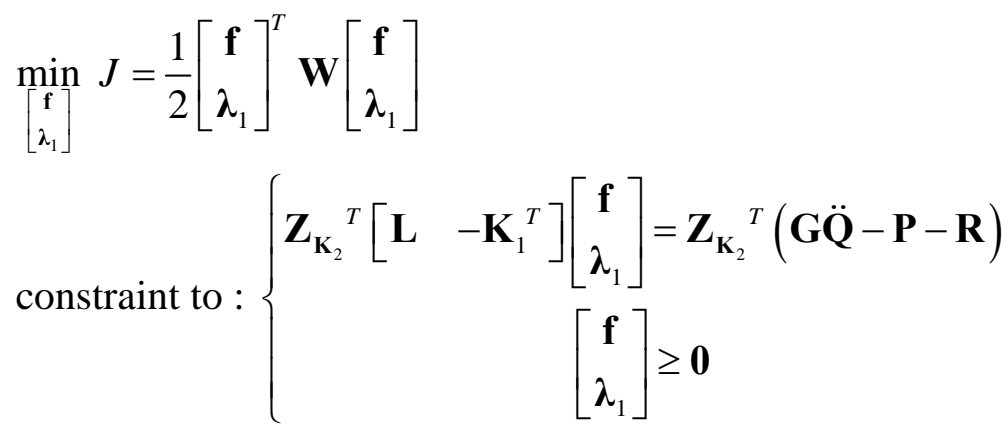

where $J$ is the objective function and $\mathbf{W}$ a diagonal matrix composed of the optimisation weights associated to the unknowns $\left[\begin{array}{ll}\mathbf{f} & \lambda_{1}\end{array}\right]^{T}$. When an optimisation weight is not null, the associated force is minimised and constrained to be positive. Otherwise, when the optimisation weight is null, the associated force is only constrained to be positive. The optimisation weights used for this study are the same as in [22, 23] and have been defined through an iterative process (i.e. 1 for musculotendon forces, 1e-6 for ligament, bone and patellofemoral joint forces, 1 for hip and ankle joint forces, 2 and 4 for medial and lateral tibiofemoral joint forces, respectively).

\section{Computation of the contributions of musculo-tendon forces}

Once the musculo-tendon forces as well as the joint contact, ligament and bone forces have been estimated by optimisation, the contributions of musculotendon forces to 3D ground reaction force and moment (i.e. $\mathbf{F}_{0}^{\mathbf{R}, f^{j}}, \mathbf{M}_{0}^{\mathbf{R}, f^{j}}$ ) can be computed. For that, a parameter reduction was applied to equation (1) to cancel the Lagrange multipliers [27, 28]:

$\mathbf{Z}_{\mathbf{K}^{T}} \mathbf{G} \ddot{\mathbf{Q}}+\underbrace{\mathbf{Z}_{\mathbf{K}^{T}} \mathbf{K}^{T} \boldsymbol{\lambda}}_{\mathbf{0}}=\mathbf{Z}_{\mathbf{K}^{T}} \mathbf{R}+\mathbf{Z}_{\mathbf{K}^{T}} \mathbf{P}+\mathbf{Z}_{\mathbf{K}^{T}} \mathbf{L f}$

where $\mathbf{Z}_{\mathbf{K}^{T}}$ is the orthogonal basis of the null space of $\mathbf{K}^{T}$. Applying individual musculo-tendon forces in isolation (i.e. $\mathbf{P}=\mathbf{0}$ and all $f^{j}=0$ except one), $\mathbf{F}_{0}^{\mathbf{R}, f^{j}}, \mathbf{M}_{0}^{\mathbf{R}, f^{j}}$ were computed by a pseudo-inverse method [7, 20] (see Supplementary material). Then, to determine the contributions to joint contact, ligament and bone forces (i.e. $\lambda_{1}^{f^{j}}$ ), the partial parameter reduction used when solving the muscular redundancy problem and defined in equation (2) was applied. Proceeding as with 3D ground reaction force and moment, we applied the individual musculo-tendon forces in isolation and, knowing $\mathbf{F}_{0}^{\mathbf{R}, f^{j}}, \mathbf{M}_{0}^{\mathbf{R}, f^{j}}$ and $\ddot{\mathbf{Q}}^{f^{j}}$, contributions $\lambda_{1}^{f^{j}}$ were then computed by a pseudo-inverse method [7] (see Supplementary material).

\section{Application to normal gait}

Gait experiments were performed on one asymptomatic male subject (30 years old, $65 \mathrm{~kg}, 165 \mathrm{~cm}$ ) in the Centre National de Rééducation Fonctionnelle et 
de Réadaptation - Rehazenter of Luxembourg. The protocol was approved by the Institutional Review Board and the subject gave informed consent prior to his participation in this study.

Joint kinematics, ground reaction and muscle electromyographic (EMG) activity were recorded simultaneously during 5 gait cycles at preferred walking speed $(1.3 \pm 0.08$ m.s-1) over level ground. Fifteen skin markers were fixed on anatomical landmarks of the pelvis (i.e. the right and left anterior and posterior superior iliac spines) and the right lower limb (i.e. the great trochanter, medial and lateral epicondyles, peroneal head, anterior tibial tuberosity, medial and lateral malleoli, calcaneus, first, second and fifth metatarsal heads) following the protocol proposed by Leardini et al. [30]. Their trajectories were recorded using a 10-camera optoelectronic system (OQUS, Qualisys AB, Sweden) sampled at $100 \mathrm{~Hz}$. Ground reaction forces and moments were recorded using 2 forceplates (OR6-5, AMTI, USA) sampled at $1500 \mathrm{~Hz}$. The EMG activity of 8 right muscles (i.e. tibialis anterior, soleus, gastrocnemius medialis, vastus medialis, rectus femoris, semitendinosus, gluteus medius and gluteus maximus) was collected with a 16channel wireless electromyographic system (DTS clinic, Noraxon, USA) sampled at $1500 \mathrm{~Hz}$. The EMG surface electrodes were placed following the recommended standard of the Surface EMG for a Non-Invasive Assessment of Muscles (SENIAM) project [31].

All data were then imported under Matlab R2011b using the Biomechanics ToolKit (BTK) [32]. Kinematic curves were interpolated when necessary using a cubic spline interpolation and smoothed by a 4th-order lowpass Butterworth filter with a cutoff frequency of $6 \mathrm{~Hz}$. Similarly, kinetic curves were smoothed by a 4thorder lowpass Butterworth filter with a cutoff frequency of $10 \mathrm{~Hz}$. Raw EMG signals were first high-pass filtered at $30 \mathrm{~Hz}$ cutoff frequency to reduce motion artefacts. Then the signals were full-wave rectified and EMG envelopes were obtained by a 4th-order low-pass Butterworth filter, applied in forward and backward directions, at $6 \mathrm{~Hz}$ cutoff frequency. Finally, all data were time-normalised to a 100\% gait cycle.

\section{Overall quality of the estimations}

To assess the validity of musculo-tendon force estimations, the coefficient of concordance proposed by Dickerson et al. [33], and recently extended to the gait analysis by Giroux et al. [34], was applied. Briefly, this method uses active/inactive state concordance between the estimated musculo-tendon forces and the EMG envelopes to compute a coefficient of concordance defined as the percentage of concordance elements. For this study, muscles were defined active when the mean value was above 20 percent of the maximum of the estimated musculo-tendon force and of the EMG envelope, respectively [35] during each of the 7 gait phases [36].

In addition, our results obtained during the stance phase of gait on 1) the superior-inferior component of 3D hip contact force, 2) tibiofemoral total contact force, 3) and the vertical component of 3D ground reaction force, were compared to the individual muscle contributions reported in the literature. The aim was to assess whether the present model and method are able to replicate them. 


\section{Analysis of the contributions of musculo-tendon forces}

Previously unreported contributions from each musculo-tendon force to 3D ground reaction moment, as well as to other joint contact, ligament and bone forces, are presented in Results. Contributions previously reported in the literature (and available as figures in Supplementary material) are also considered in the present analysis.

When muscles were modelled by several bundles and units, the results were pooled (i.e. contributions were summed up) to analyse the aforementioned 38 muscles. All the results were averaged over the 5 gait cycles and only the mean curves are provided here. The muscle groups that were the 6 major positive contributors and the 6 major negative contributors to the investigated force are listed in the figures, with their contributions. However, for readability, only muscle groups whose maximum contribution is higher than $10 \%$ of the maximum value of the investigated force are reported.

\section{Results}

\section{Overall quality of the estimations}

The concordance between the estimated musculo-tendon forces and the recorded EMG activities was good, i.e. the averaged concordance across the 5 gait cycles was $68.6 \%$ with a standard deviation of $4.7 \%$. Moreover, the present model and method applied to the present gait data were able to replicate findings in the recent literature concerning the patterns and distributions of the contributions of musculo-tendon forces to the superior-inferior component of 3D hip contact force [16], tibiofemoral total contact force [14] and the vertical component of 3D ground reaction force [7] during the stance phase of gait (Fig. 1).

Fig. 1 - Comparison between mean estimated ( $1^{\text {st }}$ column) and literature $\left(2^{\text {nd }}\right.$ column) forces expressed in body weight (BW) of a selection of contributors to the vertical component of 3D ground reaction force ( $1^{\text {st }}$ line), tibiofemoral total contact force $\left(2^{\text {nd }}\right.$ line) and the superior-inferior component of 3D hip contact force ( $3^{\text {rd }}$ line) during a gait cycle. Literature forces are reproduced respectively from [7], [14], and [16]. The digital version of the manuscript contains full details with coloured curves.

Concerning the superior-inferior component of 3D hip contact force, the maximum magnitude of the force was similar to that reported by Correa et al. [16]. The contributions of gluteus maximus and gluteus medius observed by Correa et al. [16] were reproduced. However, in our estimations, the rectus femoris played a greater role. Concerning tibiofemoral total contact force, the magnitudes of the first and second peaks of force were slightly higher than those reported in the recent literature based on implant measurements [37] (i.e. between 2 body weight (BW) and 3 BW). The patterns of the set of contributors defined by Sritharan et al. [14] (i.e. rectus femoris, vastii, hamstrings, biceps femoris short head, gastrocnemii) were reproduced. However, a marked difference was observed for the hamstrings, which made a much lower contribution in our estimations. Concerning the vertical component of 3D ground reaction force, the 5 major contributors observed by Lin 
et al. [7] were confirmed, and formed two groups linked respectively to the first (i.e. gluteus maximus, gluteus medius, vastii) and the second (i.e. soleus, gastrocnemii) peak of force.

\section{Contributions of musculo-tendon forces to ground reaction}

The contributions of musculo-tendon forces to the vertical, fore-aft and medial-lateral components of 3D ground reaction force have already been reported in the literature [2, 3, 5, 6], and figures are provided as Supplementary material. Our results concerning the contributions to the vertical component of 3D ground reaction force have already been compared to the literature in the section Overall quality of the estimations. Briefly, these results indicate that the gluteus maximus, gluteus medius, vastii, soleus and gastrocnemii are the major contributors to the vertical, fore-aft and medial-lateral components of the 3D ground reaction force.

The contributions of musculo-tendon forces to "free torque" (i.e. the vertical component of the 3D ground reaction moment computed at the CoP, which is the only non-null component) are reported in Fig. 2. This free torque is mainly internal (i.e. internal rotation moment) during the stance phase. Muscles spanning both proximal and distal joints (e.g. soleus, gemellus, gluteus maximus) contribute to an internal rotation moment, while it is mainly hip-spanning and knee-spanning muscles (e.g. gluteus medius) that contribute to an external rotation moment.

Fig. 2 - Free torque (normalised in body weight BW and leg length LL, grey area in the figure) during a gait cycle, with the 6 major positive and negative contributions to this component of 3D ground reaction moment. Only muscle groups whose maximum contribution is higher than $10 \%$ of the maximum value of the investigated force are reported. These contributors are listed on the right. The digital version of the manuscript contains full details with coloured curves.

\section{Contributions of musculo-tendon forces to joint contact forces}

The contributions of musculo-tendon forces to 3D hip contact force have already been reported in the literature [38] and figures are provided as Supplementary material. The pattern of each component of 3D hip contact force shows 2 peaks during stance at $15 \%$ and $50 \%$ of the gait cycle, and 1 peak during swing at $85 \%$ of the gait cycle. Our results concerning the contributions to the superior-inferior component of 3D hip contact force have already been compared to the literature in the section Overall quality of the estimations. The components of 3D contact force for the hip anterior-posterior contacts are often anterior; for the hip medial-lateral contacts, they are always lateral, and in both cases they are mainly due to the contributions of the gluteus medius and the quadratus femoris.

The contributions of musculo-tendon forces to tibiofemoral total contact force as well as medial and lateral contact forces have already been reported in the literature [14] and figures are provided as Supplementary material. The pattern of total, medial and lateral contact forces shows 2 peaks during stance near $15 \%$ and $45 \%$ of the gait cycle. Our results concerning the contributions to the tibiofemoral total contact force have already been compared to the literature in the section Overall quality of the estimations. For both tibiofemoral medial and lateral contact forces, the vastii and gastrocnemii contribute respectively to the first and the second peak of force. Moreover, the gluteus medius increases medial contact force and decreases lateral contact force, while the soleus acts inversely. Not surprisingly, 
knee-spanning muscles having an insertion on the medial side of the tibia increase medial contact force and decrease lateral contact force (i.e. sartorius, gracilis, adductors).

The pattern of each component of 3D patellofemoral contact force (Fig. 3) shows 2 peaks during stance near $15 \%$ and $55 \%$ of the gait cycle, and 1 peak during swing at $85 \%$ of the gait cycle. The magnitude of the first peak is always almost twice as high as that of the second peak, while the third peak is smaller than the two others. Not surprisingly, only the vastii and rectus femoris contribute to 3D patellofemoral contact force. All these muscles contribute to the first peak of force, the second peak mainly results from the action of the rectus femoris, and the third peak results from the action of the vastii.

Fig. 3 - Components of 3D patellofemoral contact force (expressed in body weight BW, grey area in the figure) during a gait cycle, with the 6 major positive and negative contributions to these forces. Only muscle groups whose maximum contribution is higher than $10 \%$ of the maximum value of the investigated force are reported. These contributors are listed on the right. The digital version of the manuscript contains full details with coloured curves.

The contributions of musculo-tendon forces to 3D ankle contact force have been partially reported in the literature [2] and figures for the superior-inferior component of 3D ankle contact force are provided as Supplementary material. The pattern of each component of 3D ankle contact force shows 2 peaks during stance near $10 \%$ and $45 \%$ of the gait cycle, and 1 peak during swing near $85 \%$ of the gait cycle. Briefly, concerning the superior-inferior component of 3D ankle contact force, the second peak of force has a much higher amplitude than the first peak, mainly caused by the action of ankle-spanning muscles (e.g. peroneii, soleus, gastrocnemii), as well as the action of non-ankle-spanning muscles (e.g. gluteus medius, sartorius, gemellus). The third peak results from the action of peroneii, and vastii. For the anterior-posterior and medial-lateral components of 3D ankle contact force (Fig. 4), maximum magnitudes are approximately $-3.8 \mathrm{BW}$ and $-0.6 \mathrm{BW}$ respectively. For the anterior-posterior component of 3D ankle contact force, the major contributors are the soleus and the peroneii, while the medial-lateral component of the 3D ankle contact force relies almost entirely on the action of the peroneii.

Fig. 4 - Anterior-posterior and medial-lateral components of 3D ankle contact force (expressed in body weight BW, grey area in the figure) during a gait cycle, with the 6 major positive and negative contributions to these forces. Only muscle groups whose maximum contribution is higher than $10 \%$ of the maximum value of the investigated force are reported. These contributors are listed on the right. The digital version of the manuscript contains full details with coloured curves.

\section{Contributions of musculo-tendon forces to ligament forces}

The pattern of the estimated anterior cruciate ligament (ACL) force (Fig. 5) shows two peaks near $10 \%$ and $50 \%$ of the gait cycle, with a magnitude of $0.4 \mathrm{BW}$ in traction. While analysing the contributions of musculo-tendon forces to ACL force is complex, involving both knee-spanning and non-knee-spanning muscles, the first peak of force seems to be mainly generated by the vastii, and the second peak of force by the soleus. 
Fig. 5 - Anterior and posterior cruciate ligaments ( $A C L$ and $P C L)$ and patellar tendon forces (expressed in body weight BW, grey area in the figure) during a gait cycle, with the 6 major positive and negative contributions to these forces. Only muscle groups whose maximum contribution is higher than $10 \%$ of the maximum value of the investigated force are reported. These contributors are listed on the right. The digital version of the manuscript contains full details with coloured curves.

Posterior cruciate ligament (PCL) force has one peak near $45 \%$ of the gait cycle, with a magnitude of 1.4BW in traction. Here again, analysing contributions is complex, with muscles acting in opposing ways, but the peak of force seems to be mainly generated by the soleus.

The estimated patellar tendon (PT) force exhibits two peaks, near 15\% and $55 \%$ of the gait cycle, with a magnitude of $1.4 \mathrm{BW}$ in traction. As with 3D patellofemoral contact force, only the vastii and the rectus femoris contribute to the PT force. Again, both the vastii and the rectus femoris contribute to the first peak of force, while the second peak is mainly generated by the action of the rectus femoris.

For both tibia-calcaneum (TiCaL) and fibula-calcaneum (CaFiL) (Fig. 6), the contributions of ankle-spanning and non-ankle-spanning muscles result in only a short occurrence of a force in traction, respectively at $62 \%$ and $85 \%$ of the gait cycle. TiCaL force has a maximum magnitude of $0.6 \mathrm{BW}$ and is mainly due to the action of the flexor hallux longus. CaFiL force has a maximum magnitude of $1.5 \mathrm{BW}$ and is due to the action of the peroneii, gluteus medius, sartorius and gracilis.

Fig. 6 - Tibia-calcaneum ligament (TiCaL) and fibula-calcaneum ligament (CaFiL) forces (expressed in body weight BW, grey area in the figure) during a gait cycle, with the 6 major positive and negative contributions to these forces. Only muscle groups whose maximum contribution is higher than $10 \%$ of the maximum value of the investigated force are reported. These contributors are listed on the right. The digital version of the manuscript contains full details with coloured curves.

\section{Contributions of musculo-tendon forces to bone forces}

The contributions of musculo-tendon forces to femur compression-traction force have already been reported in the literature [20] and figures are provided as Supplementary material. Briefly, the estimated femur compression-traction force exhibits two peaks, near $15 \%$ and $55 \%$ of the gait cycle, with a magnitude of 3.3BW in compression. These peaks rely on the contributions of muscles spanning the bone (i.e. tensor fascia lata, rectus femoris), muscles having an insertion on the femur (i.e. vastii, gluteus medius, gluteus maximus) and distant muscles (i.e. soleus).

The estimated patella compression-traction force (Fig. 7) exhibits two peaks, near $15 \%$ and $55 \%$ of the gait cycle, with a magnitude of $2.5 \mathrm{BW}$ in traction. As found for 3D patellofemoral contact force, only the vastii and the rectus femoris contribute to patella force. All these muscles contribute to the first peak of force, while the second peak mainly results from the action of the rectus femoris. All these muscles induce traction of the patella. 
Fig. 7 - Patella and tibia forces (expressed in body weight BW, grey area in the figure) during a gait cycle, with the 6 major positive and negative contributions to these forces. Only muscle groups whose maximum contribution is higher than $10 \%$ of the maximum value of the investigated force are reported. These contributors are listed on the right. The digital version of the manuscript contains full details with coloured curves.

The estimated tibia compression-traction force exhibits one peak near $45 \%$ of the gait cycle, with a magnitude of 4.5BW in compression. This peak relies on the contributions of muscles spanning the bone (i.e. gastrocnemii) and muscles having an insertion on the tibia (i.e. soleus, vastii). On the whole, the muscles only induce compression of the tibia.

\section{Discussion}

The aim of this study was to fill the gaps in the current literature by providing a comprehensive investigation of individual muscle contributions both to ground reaction and to joint contact, ligament and bone forces during normal gait. A 3D musculoskeletal model of the lower limb was exploited via a static optimisation method [22, 23]. Based on this musculoskeletal model and on the computational framework adapted from the work of Lin et al. [7], we report a large set of muscle contributions both herein and as Supplementary material.

\section{Overall quality of the estimations}

The 3D musculoskeletal model used in this study was previously validated with hip and knee instrumented prosthesis measurements [22, 38]. Nevertheless, on the whole, joint contact forces seem overestimated in our results compared to the recent literature. The superior-inferior component of 3D hip contact force found here is as high as $4.8 \mathrm{BW}$, against $3.8 \mathrm{BW}$ in the results of Correa et al. [16]. Tibiofemoral total contact force is as high as $3.7 \mathrm{BW}$, against 3.2BW in the results of Sritharan et al. [14]. This is not surprising, however, since we used a generic model. Indeed, by comparing the tibiofemoral contact forces measured by instrumented implants and estimated through a model, Fregly et al. [39] observed that the use of a generic geometry tends to overestimate these forces, in particular the tibiofemoral medial contact force [40]. Subject-specific versions of the present model may thus yield lower joint contact force estimations. For musculo-tendon forces, concordance with EMG activity was $68.6 \%$, a better result than that previously reported with this musculoskeletal model [22].

Even though different models and different datasets were used, our musculo-tendon force contribution results were close to those reported in the recent literature for the superior-inferior component of 3D hip contact force [16], tibiofemoral total contact force [14] and the vertical component of 3D ground reaction force [7]. Maximum magnitudes naturally vary, but patterns are similar, as is force distribution among muscles or groups of muscles. The most significant difference concerns the contribution of hamstrings to tibiofemoral total contact force. However, this contribution seems to be generated by a peak of force at joint contact level in [14] that was not reproduced in our study. 


\section{Contributions of musculo-tendon forces to ground reaction}

Our findings on the contributions of musculo-tendon forces to 3D ground reaction force are consistent with the recent literature [2, 3, 5-7]. Vertical support is mainly generated by the vastii, gluteus medius, gluteus maximus and tensor fascia lata during the first half of stance, and by the soleus and gastronemii during the second half of stance. Moreover, the vastii and gluteus medius decelerate the body during the first half of stance, while the soleus and gastrocnemii, as well as the peroneii, propel it forward during the second half of stance. The gluteus medius contributes to a medial force throughout the gait cycle. This action is counterbalanced by the gluteus maximus and vastii during the first half of stance, and by the quadratus femoris, adductors and gracilis during the second half of stance.

Concerning the contributions of musculo-tendon forces to free torque, the internal-external rotator muscles play a key role, but the position of the CoP also crucially affects the contribution of the ankle-spanning muscles. The gemellus and gluteus maximus are both external rotators of the hip likely to induce an external rotation moment at the $\mathrm{CoP}$ and thus an internal rotation moment in ground reaction. Moreover, during normal gait, ankle dorsiflexion increases throughout the terminal stance (i.e. $30 \%$ to $50 \%$ of the gait cycle), resulting in a tibia tilt. The soleus may thus start contributing to free torque and, due to the lateral position of the CoP, may induce an external rotation moment and thus an internal rotation moment in ground reaction.

\section{Contributions of musculo-tendon forces to joint contact forces}

Concerning the hip joint, tibiofemoral joint and ankle joint, both jointspanning and non-joint-spanning muscles contribute to 3D contact force, as previously observed and explained by Sritharan et al. [14]. These authors demonstrated that muscles that do not span the investigated joint can contribute to its loading by 1) a static or 2) a dynamic propagation of the contributions of musculo-tendon forces to 3D ground reaction force throughout the limb and 3) a dynamic propagation of musculo-tendon force contributions to 3D joint moment. However, as reported by Sritharan et al. [14], contributions to each compartment of the tibiofemoral joint follow a more complex organisation. As observed by these authors, non-knee-spanning muscles contributing to the loading of a compartment often unload the other compartment. Contrary to the results of Sritharan et al. [14], we find that, while the contributions of knee-spanning muscles to each compartment can be similar (e.g. the vastii and gastrocnemii contribute to compression in both compartments), they can also be opposite (e.g. the sartorius, gracilis).

Concerning the patellofemoral joint, only the quadriceps (i.e. the rectus femoris and vastii) contributes to $3 \mathrm{D}$ contact force. This confirms that this joint acts as a pulley where the quadriceps is the rope. Whenever the quadriceps is activated, the patella is pulled into a position that is superior, posterior and medial. On the whole, the vastii are mainly responsible for the first peak of force during midstance, while the rectus femoris mainly contributes to the second peak of force during preswing. These results are directly related to the action of these muscles. During

midstance, vastii action increases to limit the knee flexion generated by the transfer of body weight onto the single limb support [36]. Moreover, during pre-swing, the 
rectus femoris action allows passive knee flexion to be restrained, for better joint control [36].

\section{Contributions of musculo-tendon forces to ligament forces}

Contributions to cruciate ligament forces were investigated for the tibiofemoral joint. The musculoskeletal model used in this study is also applicable to the force of the medial collateral ligament of the knee, but the Lagrange multiplier associated with this ligament was not included in the optimisation process [22]. Our results point to a complex organisation with many competing structures. As described in the literature [21, 41], the quadriceps acts as antagonist to the ACL and thus helps increase its force (i.e. traction of the ligament) during the stance phase. Conversely, the hamstrings do not appear to be major contributors to increasing or restraining ACL force [41]. Interestingly, some of the main antagonists to ACL force are muscles that do not cross the knee joint. The soleus in particular contributes extensively to the second peak of ACL force during terminal stance. The strong action of the soleus during this phase contributes to knee extension, allowing the femur to advance and ensuring sufficient stride length [36]. Since ACL force tends to increase during active extension (due to the quadriceps), it is not surprising that the soleus also contributes to ACL force during terminal stance. Similar results were obtained for PCL, with one peak of force estimated during terminal stance. These results do not confirm the predictions of Shelburne et al. [42], where PCL was unloaded during stance and slightly loaded during midswing. Finally, for both ACL and PCL, several muscles have a significant agonist action. As pointed out by Mokhtarzadeh et al. [21], these muscles may be of interest in a rehabilitation program for cruciate ligament injury prevention.

For the patellofemoral joint, as for 3D patellofemoral contact force, only the quadriceps contributes to patellar tendon force, bearing out the pulley analogy for this joint.

For the ankle joint, in the case of both tibia-calcaneum and fibula-calcaneum ligaments, musculo-tendon force contributions lead to equilibrium, so that ligament force is near zero during most of the gait cycle. These results are as expected, for a normal gait with a stable ankle. Actually, one of the primary roles of both tibiacalcaneum and fibula-calcaneum ligaments is to avoid excessive adductionabduction movement of the ankle [43]. Thus, when ankle mobility is restrained in the sagittal plane, these ligaments are subject to little or no pressure. Like knee ligaments, both ankle-spanning and non-ankle-spanning muscles contribute to ankle ligament forces, leading again to a complex organisational pattern.

\section{Contributions of musculo-tendon forces to bone forces}

The compression-traction forces of the femur, patella and tibia have been reported. The musculoskeletal model used in this study is also applicable to the foot segment, but the Lagrange multiplier associated with the compression force of this segment was not included in the optimisation process [22].

It appears that muscles only induce compression on the femur and tibia during stance. However, it is not only muscles spanning the bone in question, or having an insertion on it, that contribute to its compression. For example, the soleus seems to play a major role in the compression of the femur during stance. This observation clearly illustrates the interactions at play within the musculoskeletal 
system. By contributing to knee extension during terminal stance [36], the soleus keeps 3D ground reaction force, the tibia and the femur aligned, facilitating strain transmission. Moreover, contrary to what was suggested by Lu et al. [44], our results suggest that both mono- and bi-articular muscles play a major role in bone compression. While bi-articular muscles (i.e. muscles spanning the bone) may induce compression of the full bone, mono-articular muscles (i.e. muscles having an insertion on the bone) may induce compression of the distal or proximal area of the bone and unload the other areas. However, since the present model considers bones as rigid segments, the compression of one specific area is interpreted here as full bone compression. This assumption is supported by the results obtained for the femur, patella and tibia compression-traction forces, which are consistent with the results reported respectively for tibiofemoral total contact force, the anteriorposterior component of 3D patellofemoral contact force, and the superior-inferior component of ankle contact force, in terms of magnitude of force and contributions.

Results for the patella are similar to those obtained for the contributions of musculo-tendon forces to 3D patellofemoral joint contact force. Only the quadriceps contributes to the traction of this segment.

\section{Limitations}

Although our musculoskeletal model and computational framework provide insights into the interactions within the different structures of the musculoskeletal system, this study has some limitations.

First, the use of a generic model has been widely debated in the literature, and it is now generally accepted that high prediction accuracy may only be possible through extensive personalisation of model parameters $[45,46]$. The present results should thus be interpreted with caution, and not directly applied to pathological conditions. However, the level of complexity of current musculoskeletal models suggests that they can now reasonably be used as comprehension tools, to shed light on structures, functions and interactions. We believe that the contribution of our study should be seen in this context.

Second, several structures whose interaction deserves analysis are absent from our model, such as ligaments of the hip joint, other ligaments of the tibiofemoral and ankle joints. This is due to the modelling choices made in this study. The ligaments are modelled as isometric links whose number is determined by the number of degrees of freedom of the lower limb model. The forces obtained in the ligaments represent the forces required to restrain typical degrees of freedom, tibiofemoral anterior-posterior displacement for the ACL and PCL and ankle abduction-adduction for the TiCaL and CaFiL. It is interesting to note that high forces are needed in the ACL and PCL but low forces in the TiCaL and CaFiL. In the same way, the forces obtained in the bones represent the forces required to maintain segment lengths; therefore, equivalent compression from bi-articular and mono-articular muscles is found, as discussed earlier.

Third, the model only allows the contributions of musculo-tendon forces to the forces that appear in Equation 1 to be computed. It would be useful to study segmental accelerations in addition to 3D ground reaction force and moment, and joint contact, ligament and bone forces. However, using the present methodology, contributions to the acceleration of the body centre of mass [6] cannot be computed.

Fourth, only five gait cycles for one subject were analysed in this study. Yet walking speed, motor strategies, kinematic patterns may vary, even slightly, among 
subjects, impacting the magnitude, pattern and distribution of muscle contributions. However, we believe that it is already very encouraging to see that similar results can be obtained from different subjects and different models (i.e. current study vs. the models and data used by Lin et al. [7], Sritharan et al. [14] and Correa et al. [16]).

\section{Conclusion}

To conclude, the combined musculoskeletal model and computational framework presented in this study provide insights into how the different structures of the musculoskeletal system interact. The results reported in the manuscript and as Supplementary material fill gaps in the literature, providing an overview of individual muscle contributions to ground reaction and to joint contact, ligament and bone forces during normal gait. Primary contributors are shown to be the vastii, gluteus medius, soleus, rectus femoris, gemellus, quadratus femoris, gluteus maximus and adductors. Although these observations are based on a generic model, the present method can serve as a comprehension tool that could, in future, shed light both on pathologic gait patterns and on the progression of joint disorders such as osteoarthritis.

\section{Conflict of interest}

There are no conflicts of interest associated with this research.

\section{Acknowledgments}

None.

\section{References}

1. Herzog, W., Longino, D., Clark, A.: The role of muscles in joint adaptation and degeneration. Langenbecks. Arch. Surg. 388, 305-315 (2003).

2. Pandy, M.G., Andriacchi, T.P.: Muscle and joint function in human locomotion. Annu. Rev. Biomed. Eng. 12, 401-433 (2010).

3. Higginson, J.S., Zajac, F.E., Neptune, R.R., Kautz, S.A., Delp, S.L.: Muscle contributions to support during gait in an individual with poststroke hemiparesis. J. Biomech. 39, 1769-1777 (2006).

4. Shelburne, K.B., Torry, M.R., Pandy, M.G.: Contributions of muscles, 
ligaments, and the ground-reaction force to tibiofemoral joint loading during normal gait. J. Orthop. Res. 24, 1983-1990 (2006).

5. Anderson, F.C., Pandy, M.G.: Individual muscle contributions to support in normal walking. Gait Posture. 17, 159-169 (2003).

6. $\quad$ Neptune, R.R., Zajac, F.E., Kautz, S.A.: Muscle force redistributes segmental power for body progression during walking. Gait Posture. 19, 194-205 (2004).

7. Lin, Y.C., Kim, H.J., Pandy, M.G.: A computationally efficient method for assessing muscle function during human locomotion. Int. J. Numer. Methos Biomed. Eng. 27, 436-449 (2011).

8. Hamner, S.R., Delp, S.L.: Muscle contributions to fore-aft and vertical body mass center accelerations over a range of running speeds. J. Biomech. 46, 780-787 (2013).

9. Liu, M.Q., Anderson, F.C., Schwartz, M.H., Delp, S.L.: Muscle contributions to support and progression over a range of walking speeds. J. Biomech. 41, 3243-3252 (2008).

10. Caruthers, E.J., Thompson, J.A., Chaudhari, A.M.W., Schmitt, L.C., Best, T.M., Saul, K.R., Siston, R.A.: Muscle forces and their contributions to vertical and horizontal acceleration of the Center of mass during sit-tostand transfer in young, healthy adults. J. Appl. Biomech. 32, 487-503 (2016).

11. Dixon, P.C., Jansen, K., Jonkers, I., Stebbins, J., Theologis, T., Zavatsky, A.B.: Muscle contributions to centre of mass acceleration during turning gait in typically developing children: A simulation study. J. Biomech. 48, 4238-4245 (2015).

12. Neptune, R.R., McGowan, C.P.: Muscle contributions to frontal plane angular momentum during walking. J. Biomech. 49, 2975-2981 (2016).

13. Arnold, A.S., Schwartz, M.H., Thelen, D.G., Delp, S.L.: Contributions of muscles to terminal-swing knee motions vary with walking speed. J. Biomech. 40, 3660-3671 (2007).

14. Sritharan, P., Lin, Y.-C., Pandy, M.G.: Muscles that do not cross the knee contribute to the knee adduction moment and tibiofemoral compartment loading during gait. J. Orthop. Res. 30, 1586-1595 (2012). 
15. Pandy, M.G., Lin, Y.C., Kim, H.J.: Muscle coordination of mediolateral balance in normal walking. J. Biomech. 43, 2055-2064 (2010).

16. Correa, T.A., Crossley, K.M., Kim, H.J., Pandy, M.G.: Contributions of individual muscles to hip joint contact force in normal walking. $\mathrm{J}$. Biomech. 43, 1618-1622 (2010).

17. Collins, J.J., O’Connor, J.J.: Muscle-ligament interactions at the knee during walking. Proc. Inst. Mech. Eng. H. 205, 11-18 (1991).

18. Fregly, B.J., Lin, Y., Walter, J.P., Pandy, M.G., Banks, S.A., D’Lima, D.D.: Muscle and contact contributions to inverse dynamic knee loads during gait. Proc. ASME 2009 Summer Bioeng. Conf. SBC2009-206558 (2009).

19. Saxby, D.J., Modenese, L., Bryant, A.L., Gerus, P., Killen, B., Fortin, K., Wrigley, T. V., Bennell, K.L., Cicuttini, F.M., Lloyd, D.G.: Tibiofemoral contact forces during walking, running and sidestepping. Gait Posture. 49, 78-85 (2016).

20. Moissenet, F., Chèze, L., Dumas, R.: Contribution of individual musculotendon forces to the axial compression force of the femur during normal gait. Mov. Sport Sci. - Sci. Mot. 93, 63-69 (2016).

21. Mokhtarzadeh, H., Yeow, C.H., Hong Goh, J.C., Oetomo, D., Malekipour, F., Lee, P.V.S.: Contributions of the Soleus and Gastrocnemius muscles to the anterior cruciate ligament loading during single-leg landing. J. Biomech. 46, 1913-1920 (2013).

22. Moissenet, F., Chèze, L., Dumas, R.: A 3D lower limb musculoskeletal model for simultaneous estimation of musculo-tendon, joint contact, ligament and bone forces during gait. J. Biomech. 47, 50-58 (2014).

23. Moissenet, F., Chèze, L., Dumas, R.: Influence of the level of muscular redundancy on the validity of a musculoskeletal model. J. Biomech. Eng. 138, (2016).

24. Klein Horsman, M.D., Koopman, H.F.J.M., van der Helm, F.C.T., Prosé, L.P., Veeger, H.E.J.: Morphological muscle and joint parameters for musculoskeletal modelling of the lower extremity. Clin. Biomech. (Bristol, Avon). 22, 239-247 (2007).

25. van Arkel, R.J., Modenese, L., Phillips, A.T.M., Jeffers, J.R.T.: Hip abduction can prevent posterior edge loading of hip replacements. J. Orthop. Res. 31, 1172-1179 (2013). 
26. Dumas, R., Moissenet, F., Gasparutto, X., Cheze, L., Chèze, L., Cheze, L.: Influence of joint models on lower-limb musculo-tendon forces and threedimensional joint reaction forces during gait. Proc. Inst. Mech. Eng. Part $\mathrm{H}$ J. Eng. Med. 226, 146-160 (2012).

27. Moissenet, F., Chèze, L., Dumas, R.: Anatomical kinematic constraints: consequences on musculo-tendon forces and joint reactions. Multibody Syst. Dyn. 28, 125-141 (2012).

28. Garcia de Jalon, J., Bayo, E.: Kinematic and dynamic simulation of multibody systems. The real-time challenge. Springer-Verlag, New-York (1994).

29. Moissenet, F., Chèze, L., Dumas, R.: Potential of the pseudo-inverse method as a constrained static optimization for musculo-tendon forces prediction. J. Biomech. Eng. 134, 64503 (2012).

30. Leardini, A., Sawacha, Z., Paolini, G., Ingrosso, S., Nativo, R., Benedetti, M.G.: A new anatomically based protocol for gait analysis in children. Gait Posture. 26, 560-571 (2007).

31. Hermens, H.J., Freriks, B., Disselhorst-Klug, C., Rau, G.: Development of recommendations for SEMG sensors and sensor placement procedures. J. Electromyogr. Kinesiol. 10, 361-374 (2000).

32. Barre, A., Armand, S.: Biomechanical ToolKit: Open-source framework to visualize and process biomechanical data. Comput. Methods Programs Biomed. 114, 80-87 (2014).

33. Dickerson, C.R., Hughes, R.E., Chaffin, D.B.: Experimental evaluation of a computational shoulder musculoskeletal model. Clin. Biomech. (Bristol, Avon). 23, 886-894 (2008).

34. Giroux, M., Moissenet, F., Dumas, R.: EMG-based validation of musculoskeletal models for gait analysis. Comput. Methods Biomech. Biomed. Engin. 16, 152-156 (2013).

35. Pedersen, D.R., Brand, R.A., Cheng, C., Arora, J.S.: Direct comparison of muscle force predictions using linear and nonlinear programming. J. Biomech. Eng. 109, 192-199 (1987).

36. Perry, J., Burnfield, J., Perry, D.J., Burnfield, D.J.: Gait Analysis: Normal and Pathological Function. SLACK Incorporated (1992). 
37. Kinney, A.L., Besier, T.F., D’Lima, D.D., Fregly, B.J.: Update on grand challenge competition to predict in vivo knee loads. J. Biomed. Eng. 135, 210121-210124 (2013).

38. Moissenet, F., Giroux, M., Chèze, L., Dumas, R.: Validity of a musculoskeletal model using two different geometries for estimating hip contact forces during normal walking. Comput. Methods Biomech. Biomed. Engin. 18 Suppl 1, 2000-2001 (2015).

39. Fregly, B.J., Besier, T.F., Lloyd, D.G., Delp, S.L., Banks, S.A., Pandy, M.G., D’Lima, D.D.: Grand challenge competition to predict in vivo knee loads. J. Orthop. Res. 30, 503-513 (2012).

40. Gerus, P., Sartori, M., Besier, T.F., Fregly, B.J., Delp, S.L., Banks, S.A., Pandy, M.G., D’Lima, D.D., Lloyd, D.G.: Subject-specific knee joint geometry improves predictions of medial tibiofemoral contact forces. J. Biomech. 46, 2778-2786 (2013).

41. Beynnon, B.D., Fleming, B.C.: Anterior cruciate ligament strain in-vivo: a review of previous work. J. Biomech. 31, 519-525 (1998).

42. Shelburne, K.B., Pandy, M.G., Anderson, F.C., Torry, M.R.: Pattern of anterior cruciate ligament force in normal walking. J. Biomech. 37, 797805 (2004).

43. Leardini, A., O’Connor, J.J., Catani, F., Giannini, S.: The role of the passive structures in the mobility and stability of the human ankle joint: a literature review. Foot ankle Int. 21, 602-615 (2000).

44. Lu, T.W., O’Connor, J.J., Taylor, S.J., Walker, P.S.: Validation of a lower limb model with in vivo femoral forces telemetered from two subjects. J. Biomech. 31, 63-69 (1998).

45. Marra, M.A., Vanheule, V., Fluit, R., Koopman, B.H.F.J.M., Rasmussen, J., Verdonschot, N.J.J., Andersen, M.S., Fluit, R., Koopman, B.H.F.J.M., Rasmussen, J., Verdonschot, N.J.J., Andersen, M.S.: A Subject-Specific Musculoskeletal Modeling Framework to Predict in Vivo Mechanics of Total Knee Arthroplasty. J. Biomech. Eng. 137, 20904 (2014).

46. Lenaerts, G., De Groote, F., Demeulenaere, B., Mulier, M., Van der Perre, G., Spaepen, A., Jonkers, I.: Subject-specific hip geometry affects predicted hip joint contact forces during gait. J. Biomech. 41, 1243-1252 (2008). 
Our estimated contributions

Superior-inferior component of

3D hip contact force
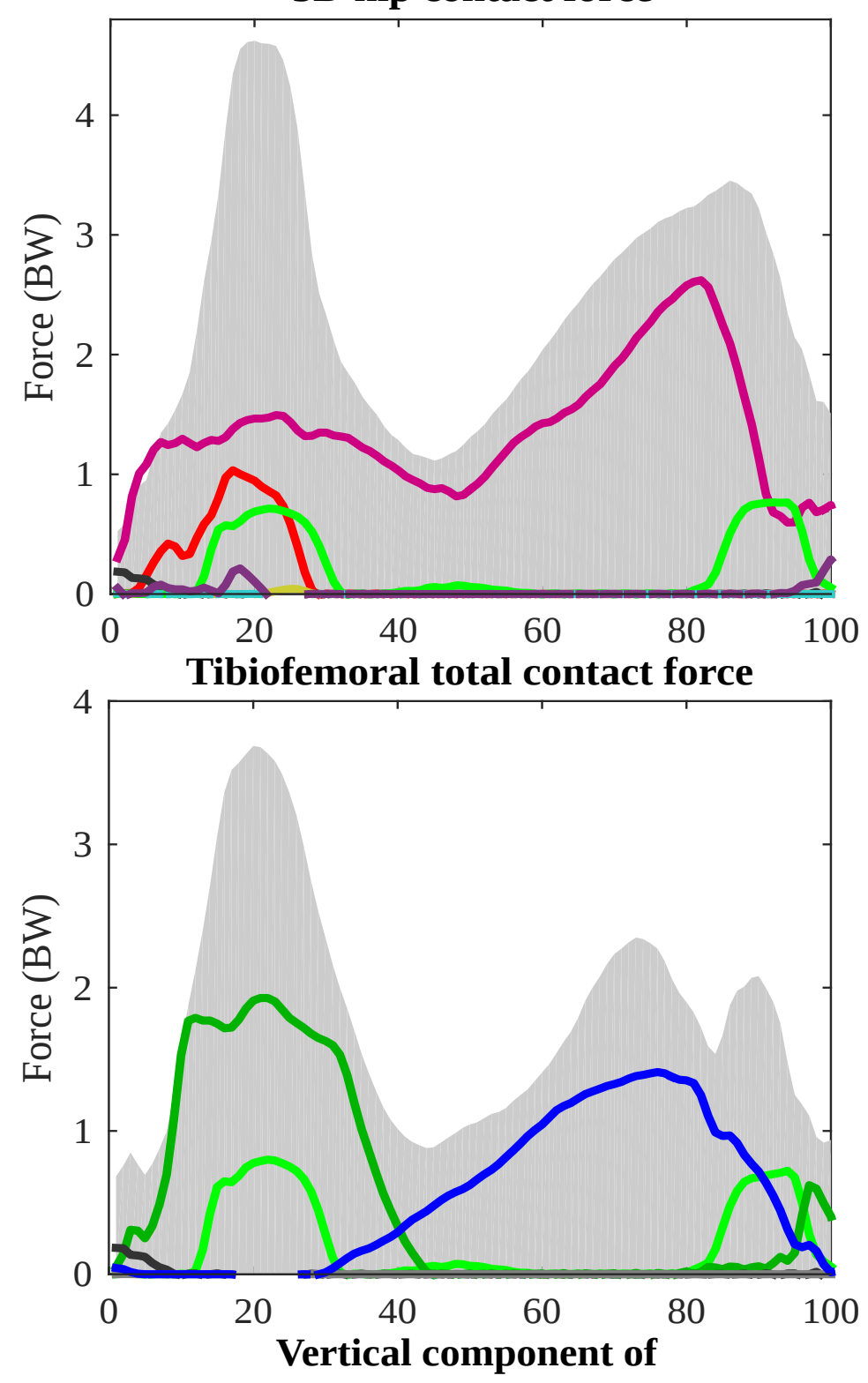

3D ground reaction force

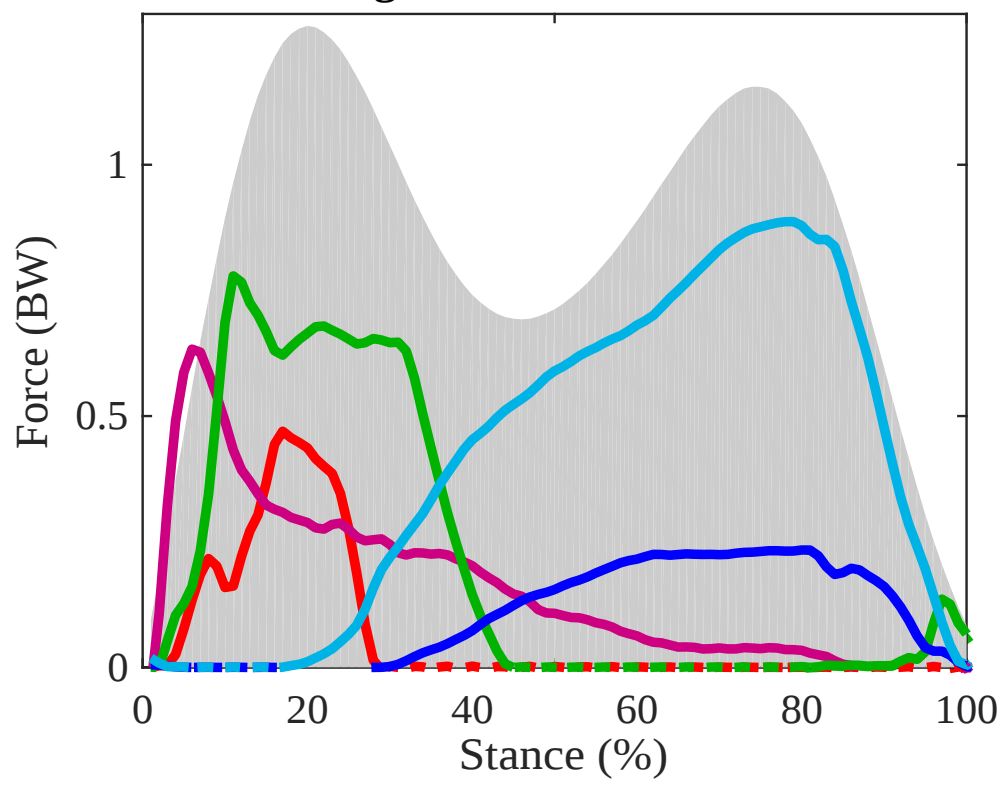

— Gluteus maximus — Piriformis

— Gluteus medius — Adductors

— Iliopsoas
Literature contributions

Superior-inferior component of

3D hip contact force
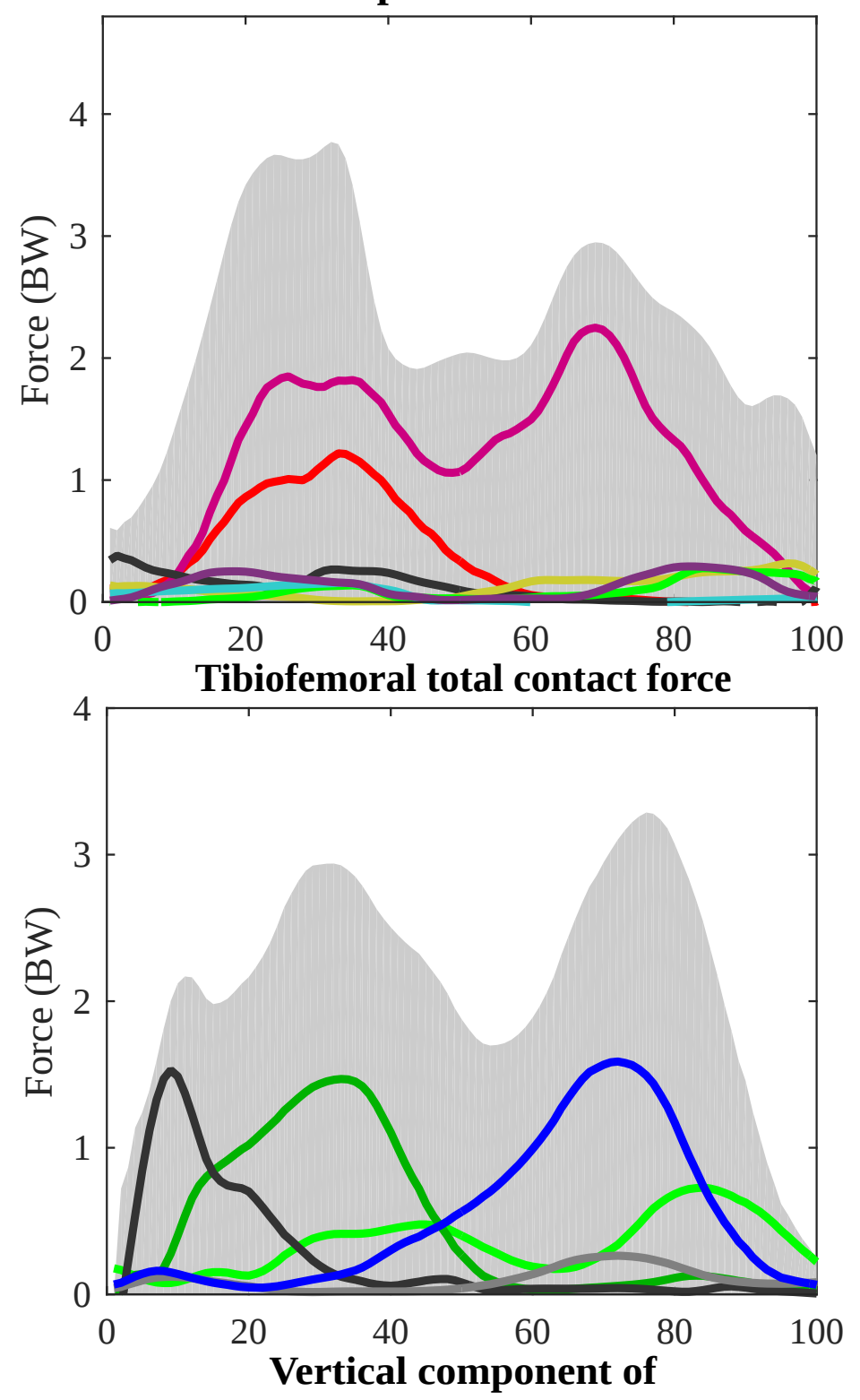

3D ground reaction force

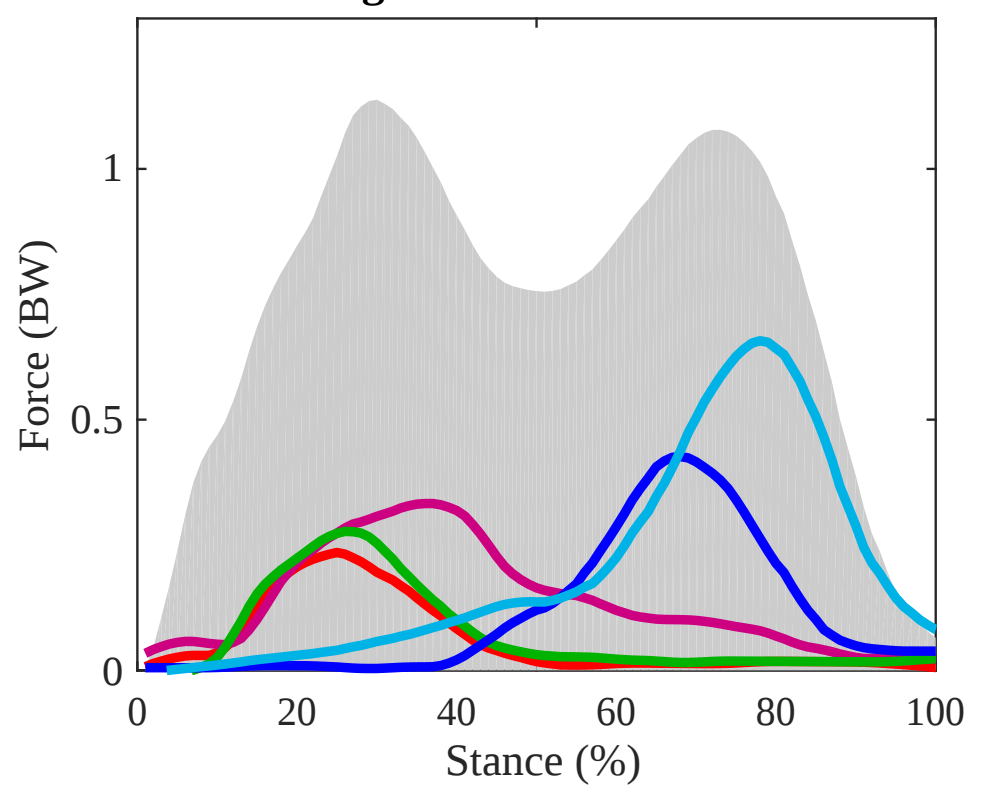




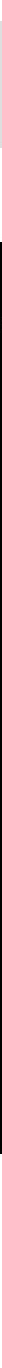




\section{Superior-inferior component of}

3D patellofemoral contact force
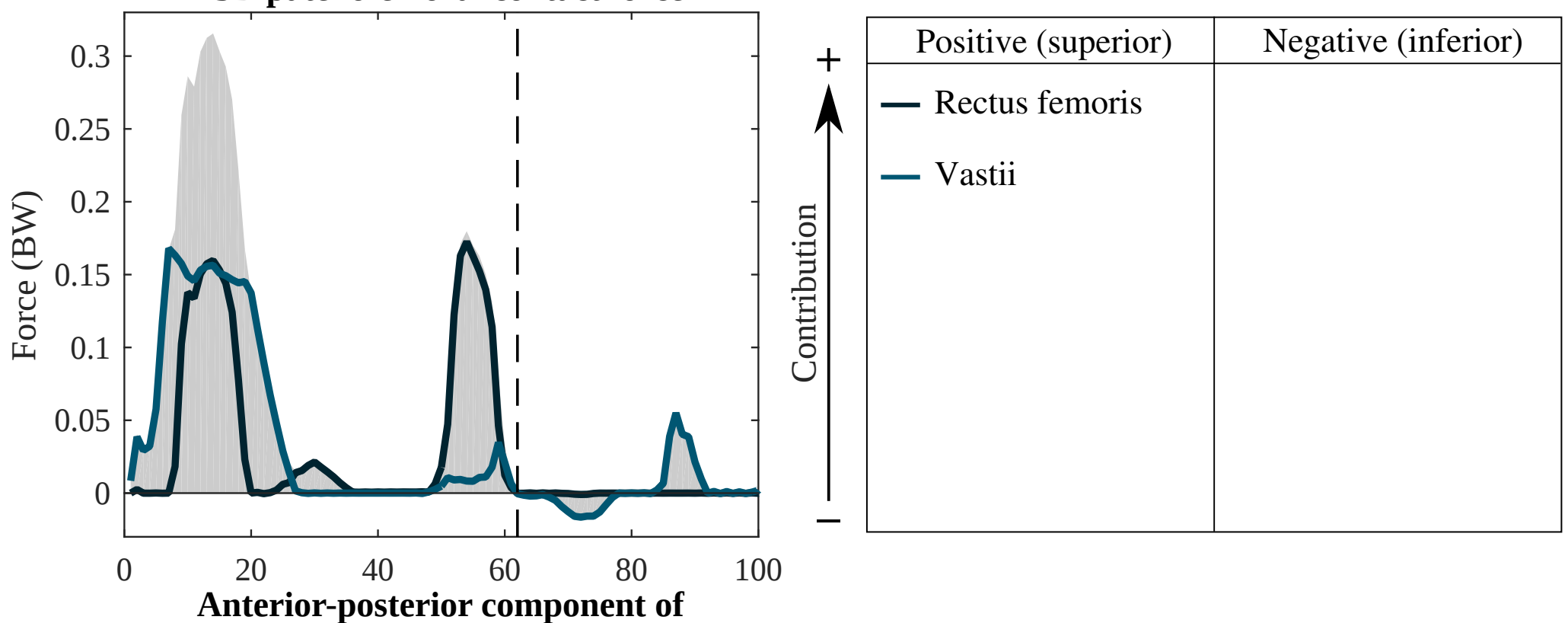

3D patellofemoral contact force
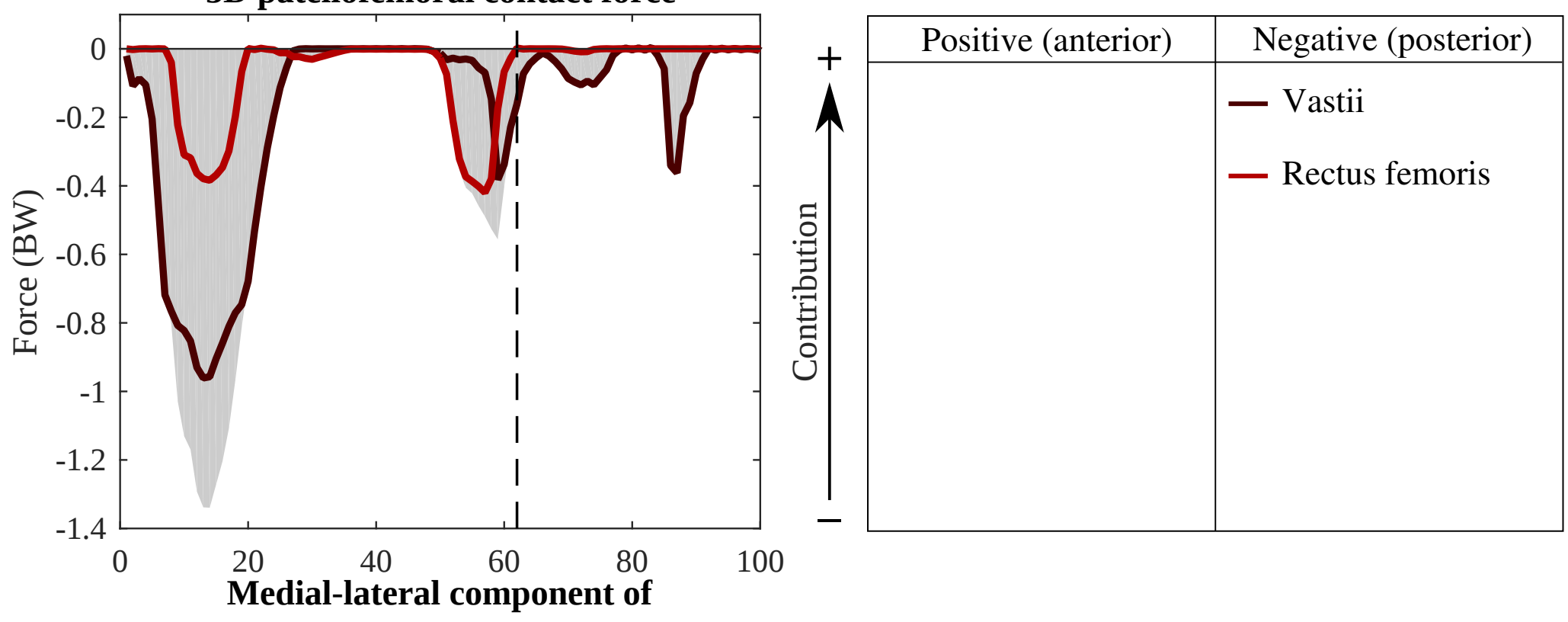

3D patellofemoral contact force
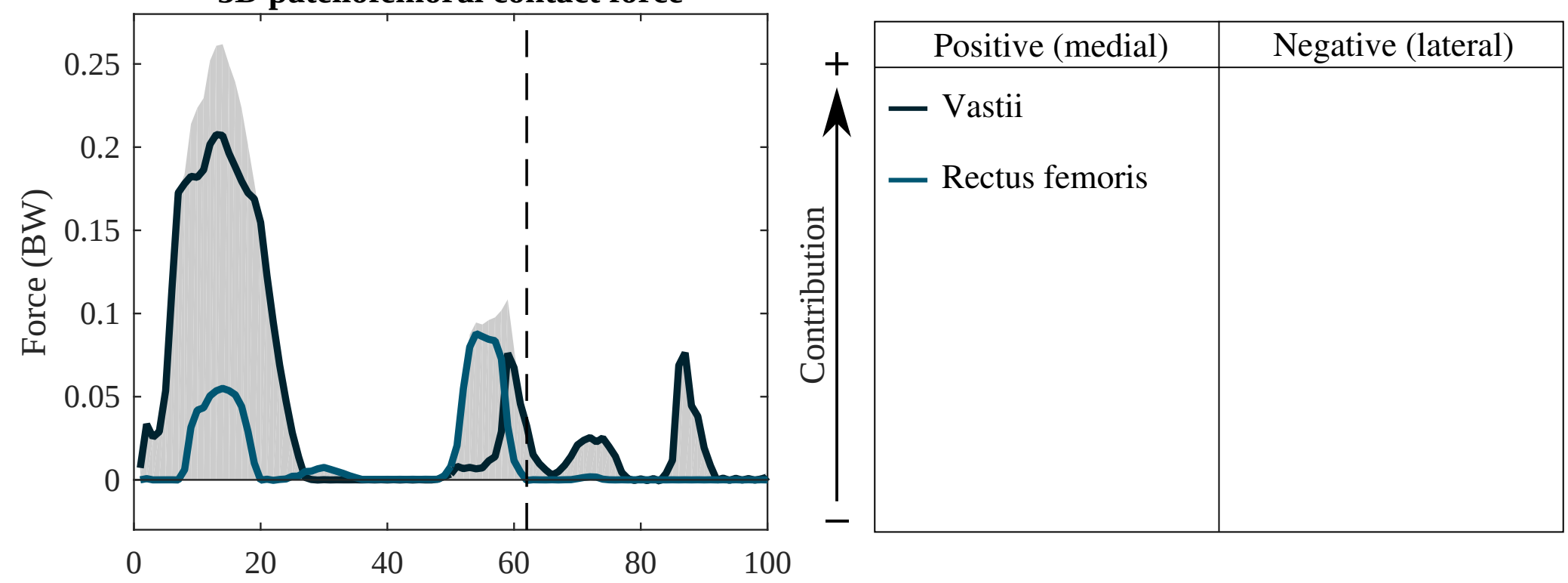

Gait cycle (\%) 


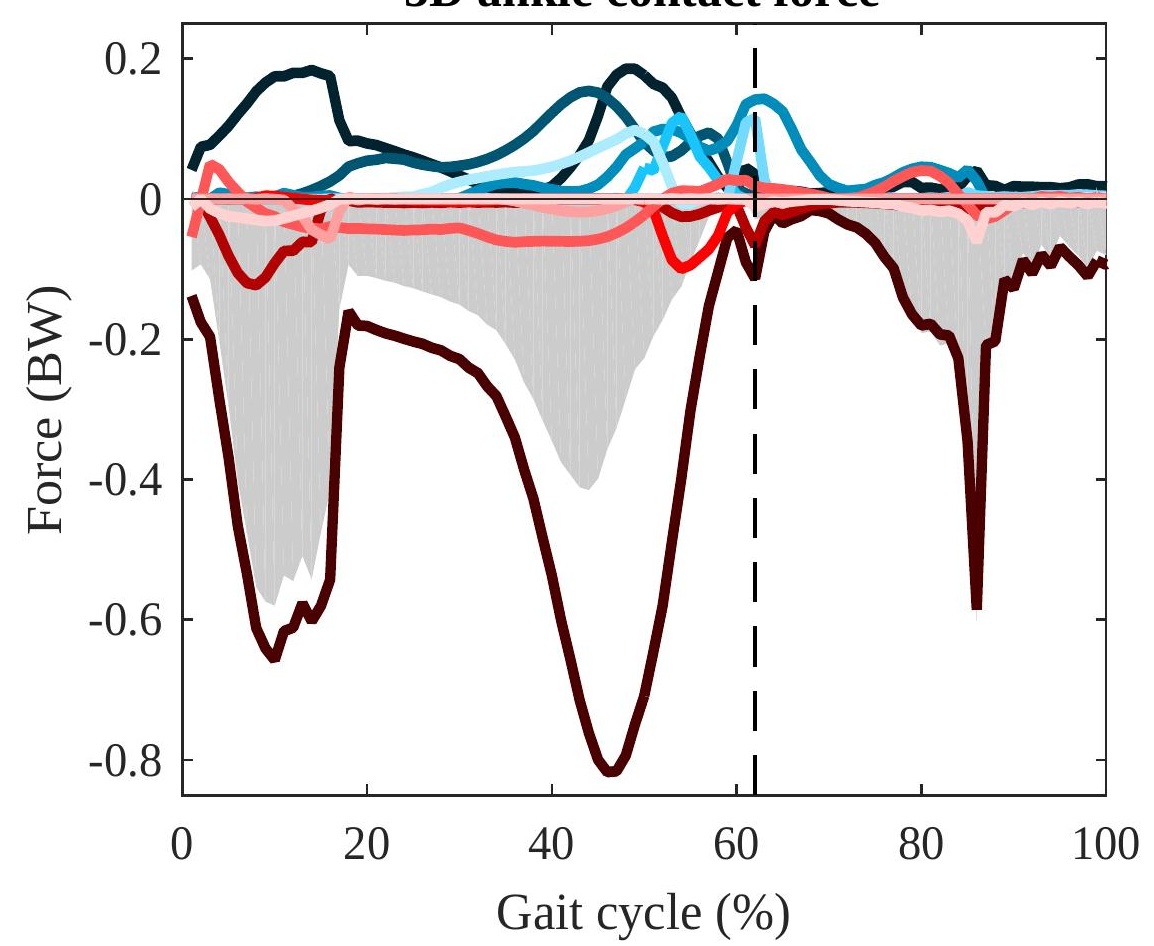

\begin{tabular}{|c|c|}
\hline Positive (anterior) & Negative (posterior) \\
\hline - Gluteus medius & - Soleus \\
\hline - Sartorius & — Peroneii \\
\hline - Adductors & — Vastii \\
\hline - Gracilis & — Gemellus \\
\hline - Quadratus femoris & $\begin{array}{l}\text { Flexor hallux } \\
\text { longus }\end{array}$ \\
\hline - Hamstrings & _ Gluteus maximus \\
\hline
\end{tabular}

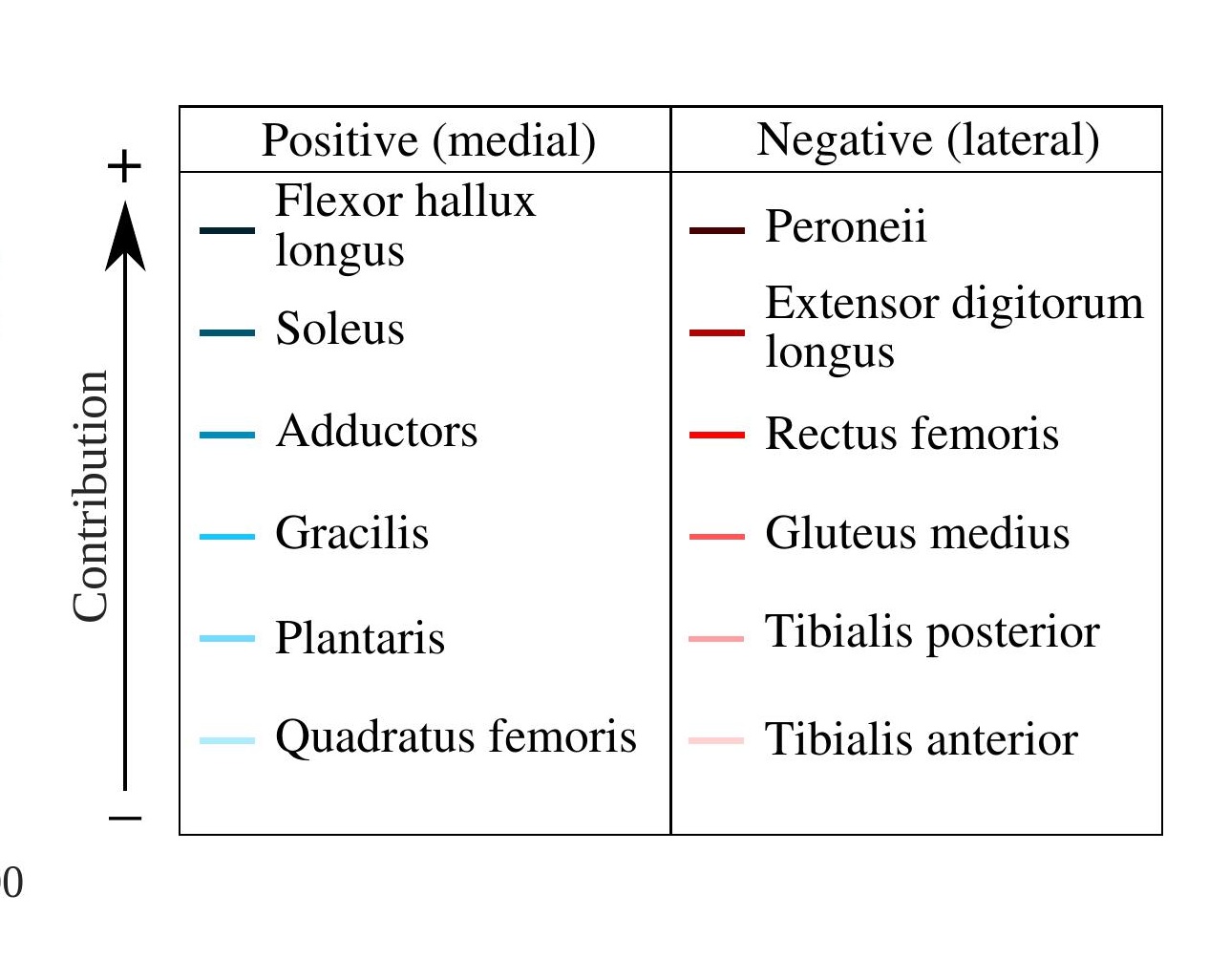

Main contributors 
Our estimated contributions

Anterior cruciate ligament force
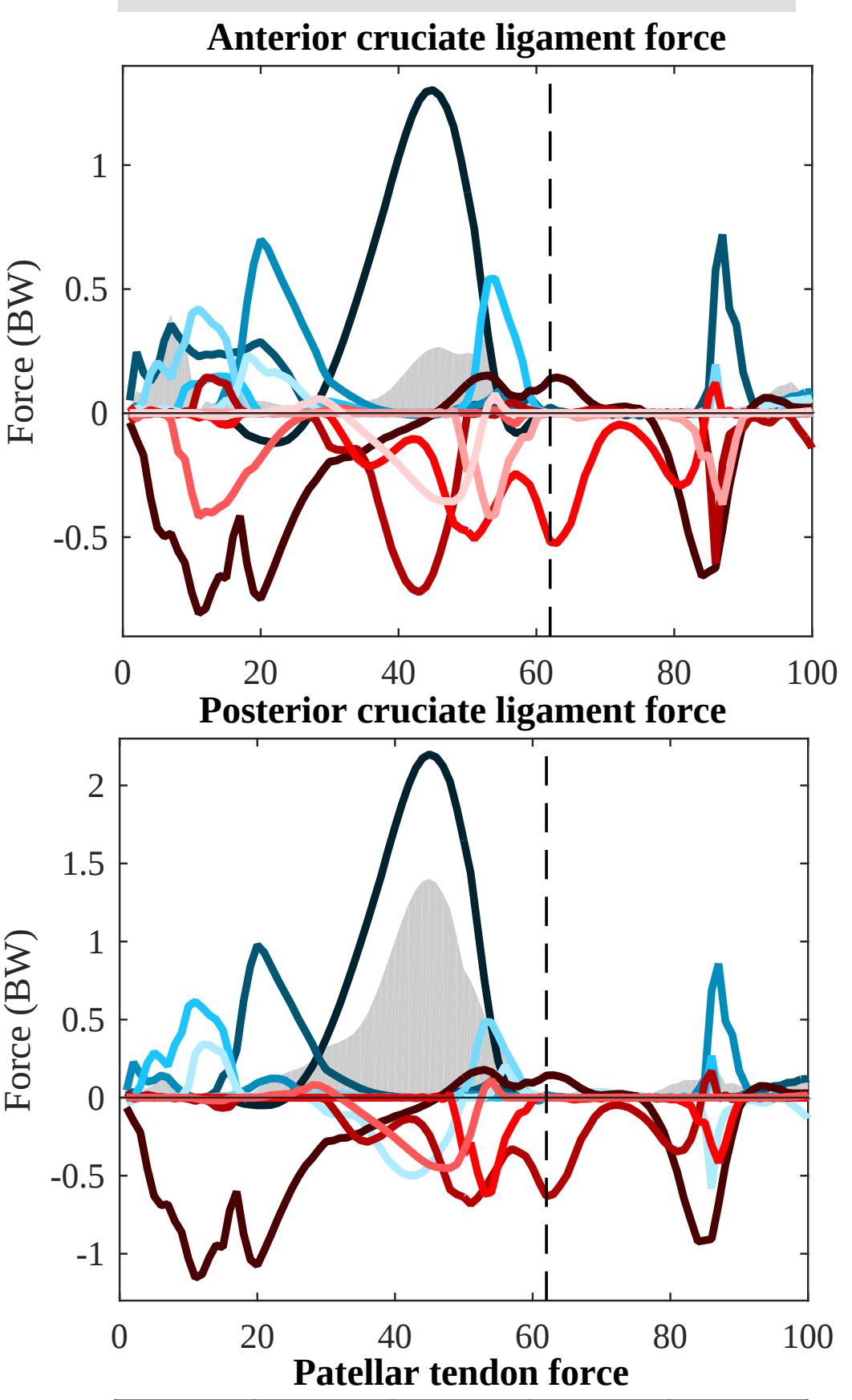

Fig. 5



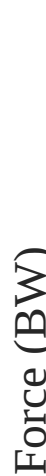

1.4
1
0.2
0.6
0.2

0

$$
0
$$

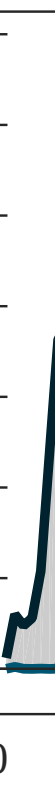

\section{Main contributors}

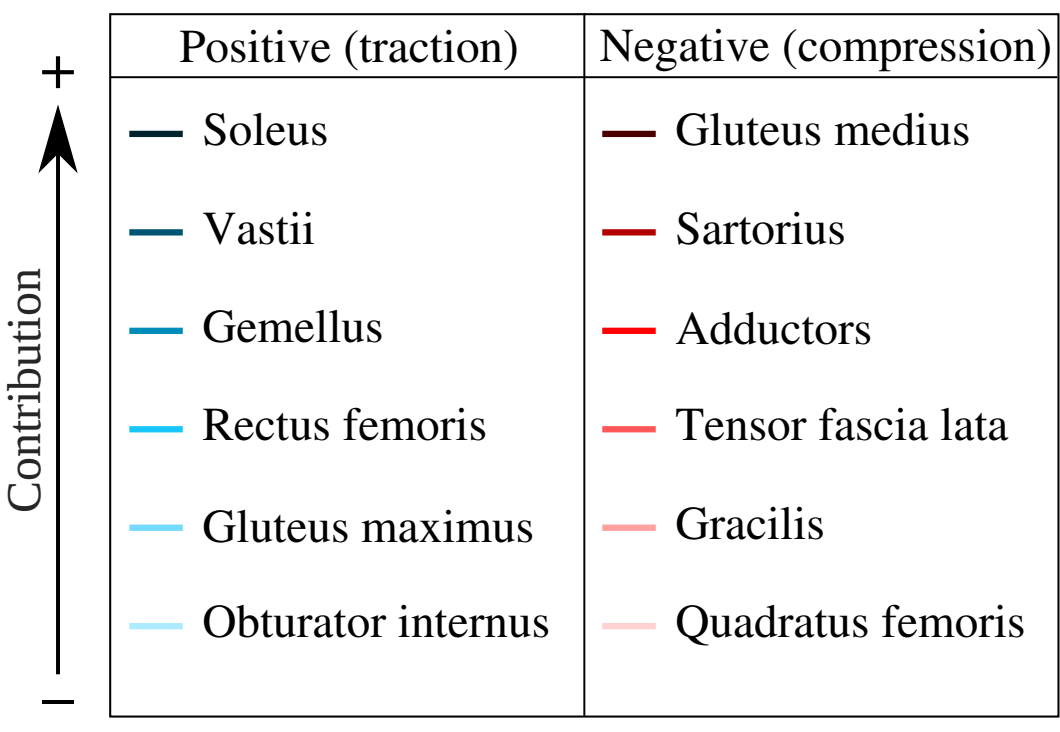

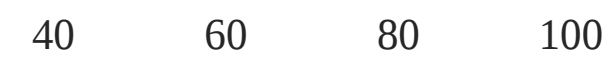

Gait cycle (\%)

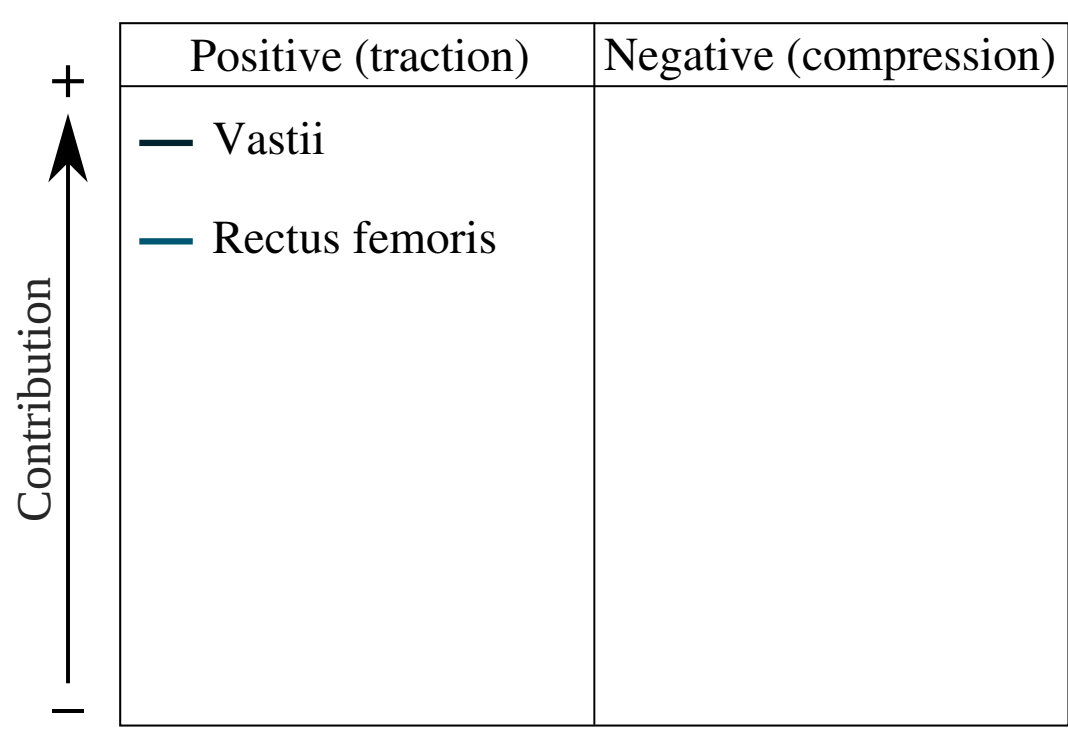

\begin{tabular}{l|l}
\hline \multicolumn{1}{c|}{ Positive (traction) } & Negative (compression) \\
\hline - Soleus & - Gluteus medius \\
- Gemellus & - Adductors \\
- Vastii & - Gracilis \\
- Gluteus maximus & - Quadratus femoris \\
- Rectus femoris & \\
- Sartorius & \\
\hline
\end{tabular}




\section{Our estimated contributions}

\section{Tibia-calcaneum ligament force}
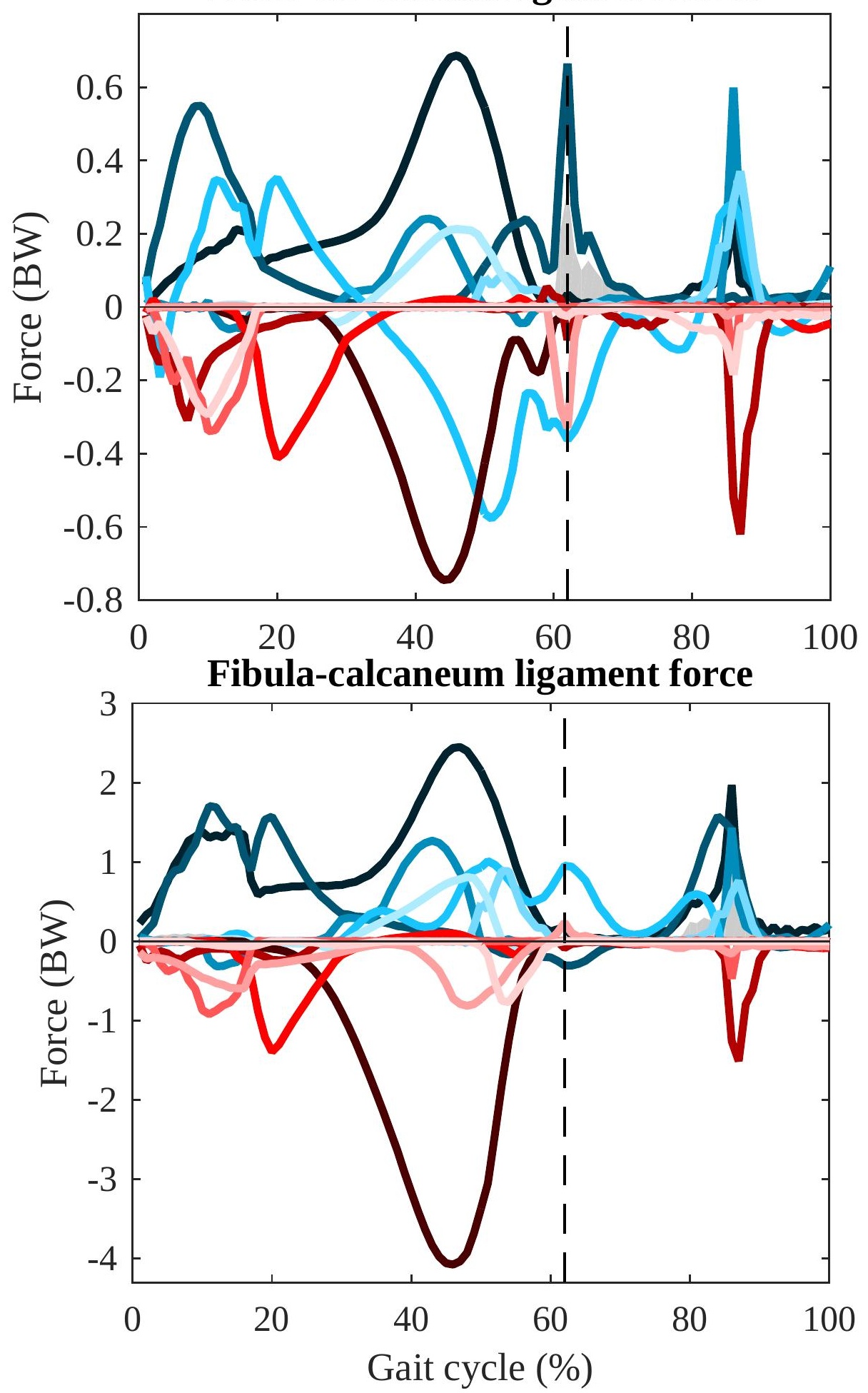

Main contributors

\begin{tabular}{|c|c|}
\hline Positive (traction) & Negative (compression) \\
\hline - Peroneii & - Soleus \\
\hline $\begin{array}{l}\text { Flexor hallux } \\
\text { longus }\end{array}$ & - Vastii \\
\hline - Sartorius & — Gemellus \\
\hline — Gluteus medius & — Gluteus maximus \\
\hline - Gracilis & - Plantaris \\
\hline - Quadratus femoris & - Tibialis anterior \\
\hline
\end{tabular}

\begin{tabular}{|c|c|}
\hline Positive (traction) & Negative (compression) \\
\hline - Peroneii & - Soleus \\
\hline — Gluteus medius & - Vastii \\
\hline — Sartorius & — Gemellus \\
\hline — Adductors & — Gluteus maximus \\
\hline - Gracilis & $\begin{array}{l}\text { Flexor hallux } \\
\text { longus }\end{array}$ \\
\hline - Quadratus femoris & - Rectus femoris \\
\hline
\end{tabular}




\section{Our estimated contributions}
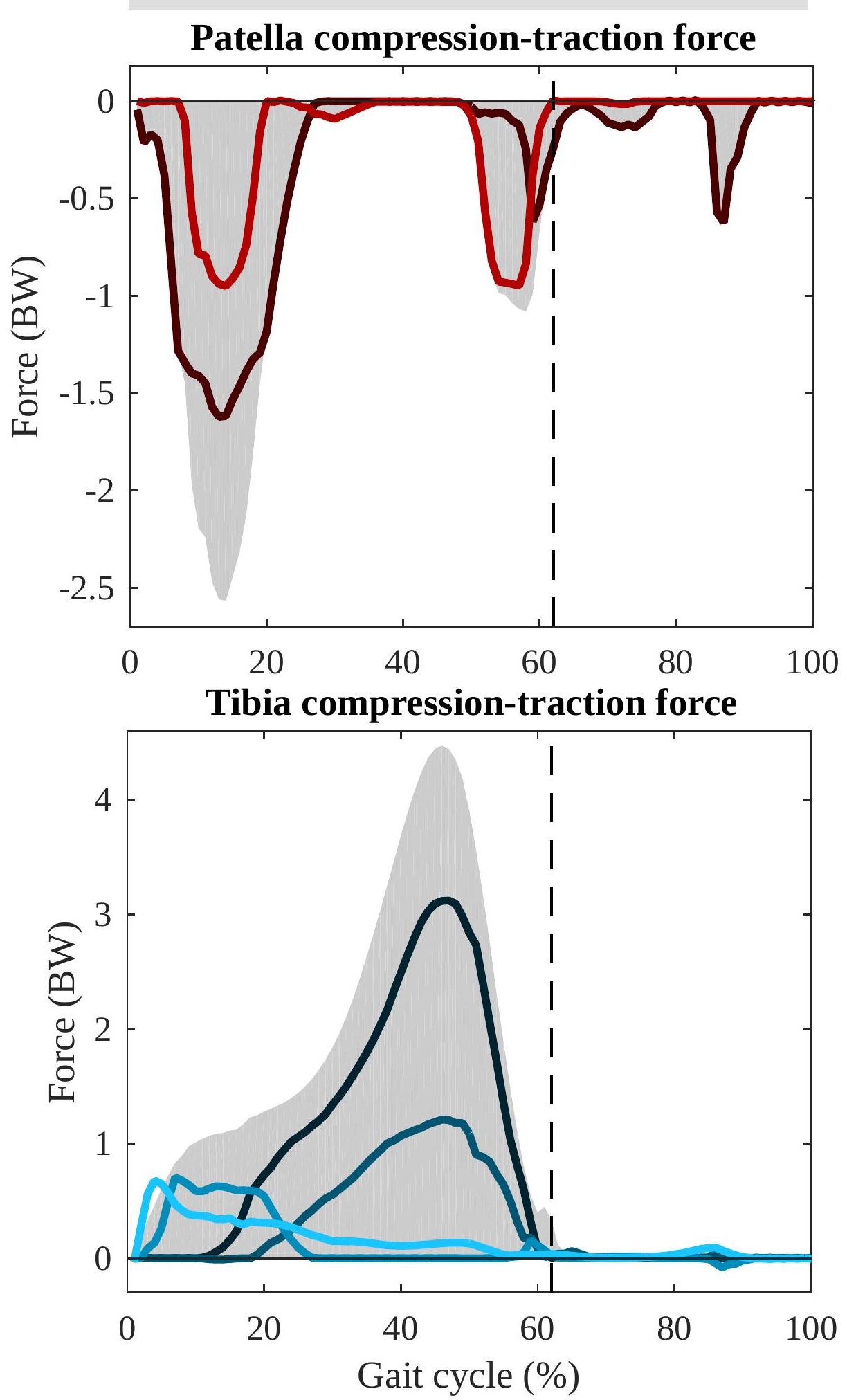
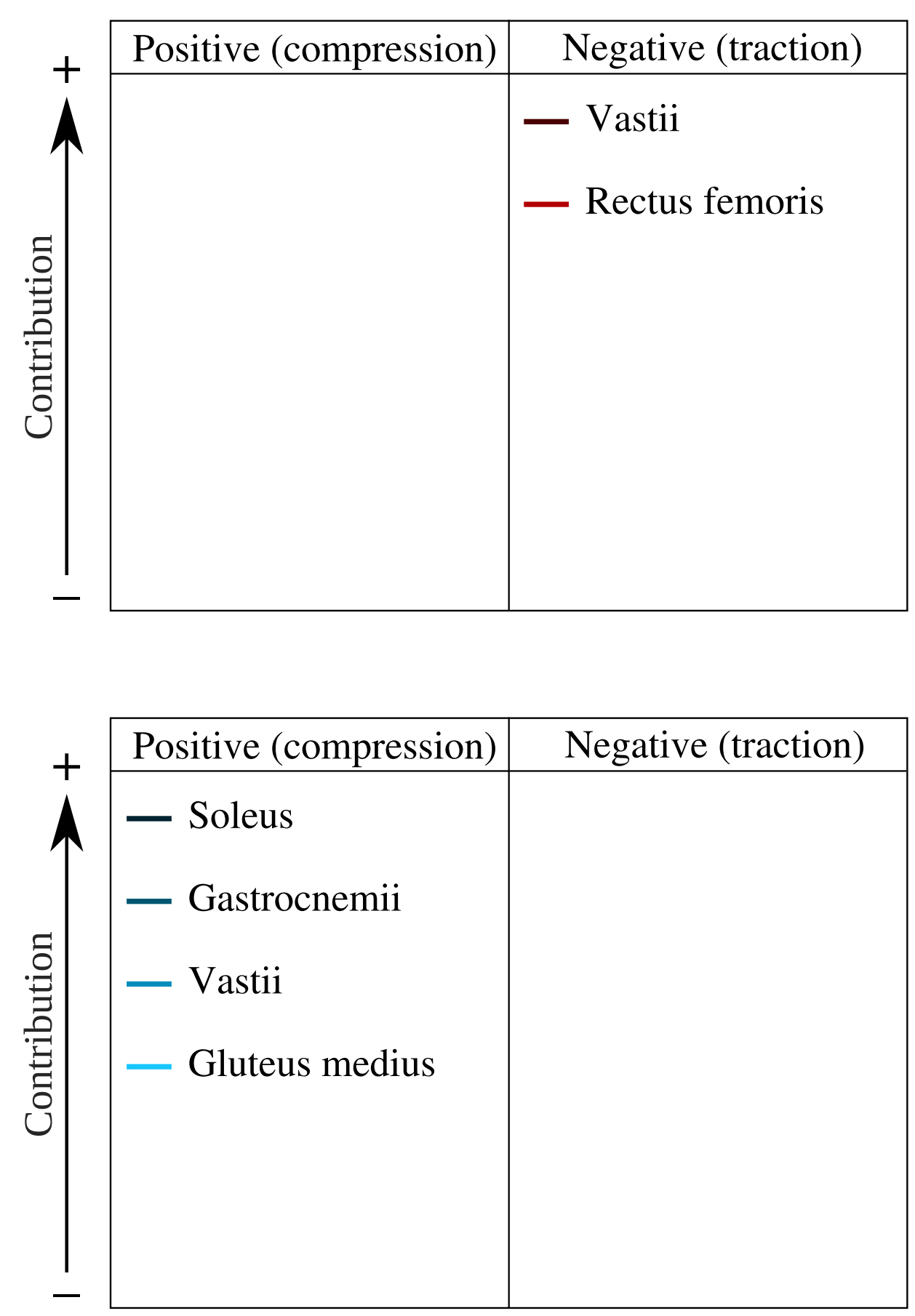

Main contributors 


\section{Supplementary material}

\section{S1. Joint kinematic models}

In the 3D lower limb musculoskeletal model (Figure S1), the hip joint was modelled by a spherical joint. The tibiofemoral joint was modelled by a parallel mechanism made of two sphere-on-plane contacts (i.e. medial and lateral) and three isometric ligaments (i.e. anterior cruciate ligament - ACL, posterior cruciate ligament - PCL and medial collateral ligament - MCL) [Feikes2003]. The patellofemoral joint was modelled by a hinge joint between the patella and the femur and an isometric ligament (i.e. the patellar tendon - PT) between the patella and the tibia [Sancisi2011]. The ankle joint was modelled by a parallel mechanism composed of a spherical joint and two isometric ligaments (between tibia and calcaneus - TiCaL and between fibula and calcaneus - CaFiL)

[DiGregorio2007].

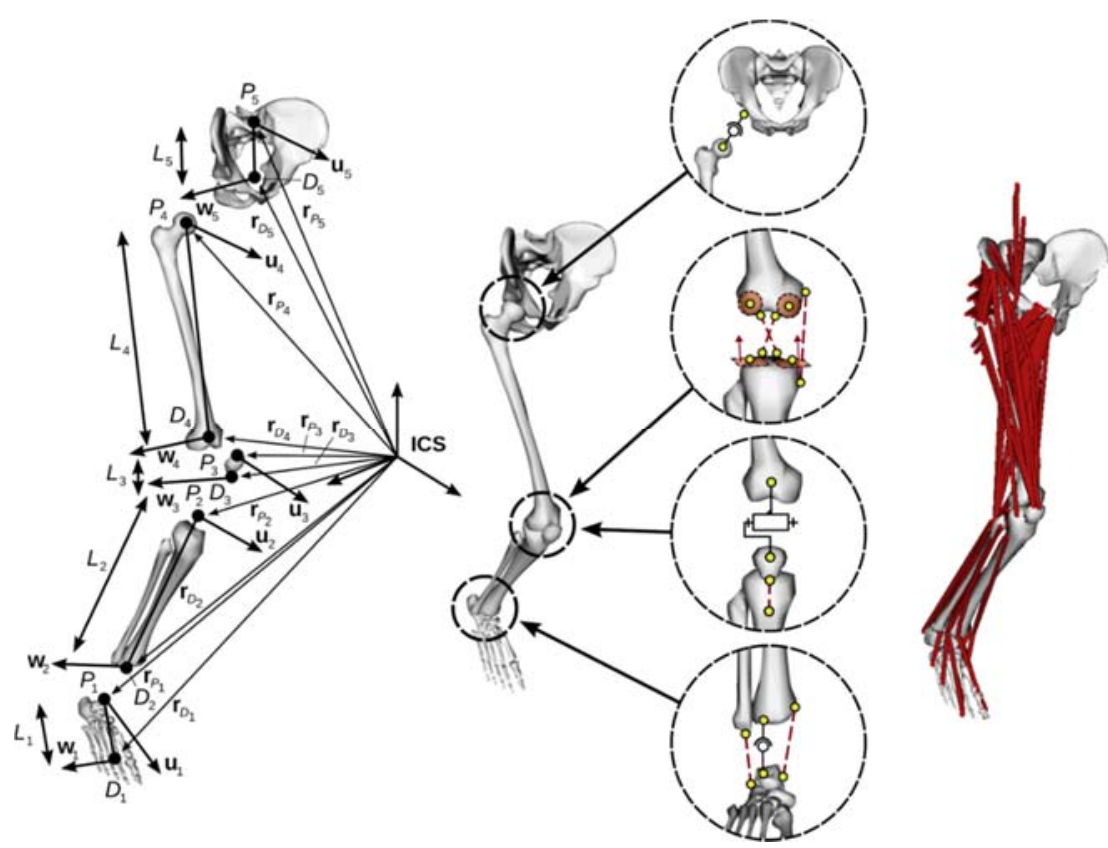

Figure S1 - 3D lower limb musculoskeletal model

These joint kinematic models are implemented as kinematics constraints. The related Lagrange multipliers correspond straightforwardly to the joint contact and ligament [Moissenet2012]. 


\section{S2. Computational framework}

\section{Introduction of 3D forces}

To perform the contribution analysis based on equations (2) and (3), the ground reaction wrench (i.e. force and moment) $\left\{\begin{array}{l}\mathbf{F}_{0}^{\mathbf{R}} \\ \mathbf{M}_{0}^{\mathbf{R}}\end{array}\right.$ at the CoP $\mathbf{r}_{P_{0}}$ and the dynamic wrenches $\left\{\begin{array}{l}\mathbf{F}_{i}^{\mathbf{G}} \\ \mathbf{M}_{i}^{\mathbf{G}}\end{array}\right.$ at the centres of mass $\mathbf{r}_{C_{i}}$ of each segment (i.e. standing for the inertia, centrifugal and Coriolis actions) need to be transformed, so as to avoid using moments in the contribution analysis [Lin2011]. Segment indices $i=0 \ldots 4$ correspond respectively to forceplate, foot, tibia/fibula, patella and femur segments.

Following the formulation of Dumas et al. [Dumas2012], the vector of generalised ground reaction $\mathbf{R}$, as it appears in Equations 1, 2, and 3, is:

$$
\mathbf{R}=\left[\begin{array}{c}
{\left[\mathbf{N}_{1}^{P_{1}}\right]^{T} \mathbf{F}_{0}^{\mathbf{R}}+\left[\mathbf{N}_{1}^{*}\right]^{T}\left(\mathbf{M}_{0}^{\mathbf{R}}+\left(\mathbf{r}_{P_{0}}-\mathbf{r}_{P_{1}}\right) \times \mathbf{F}_{0}^{\mathbf{R}}\right)} \\
\mathbf{0}_{12 \times 1} \\
\mathbf{0}_{12 \times 1} \\
\mathbf{0}_{12 \times 1}
\end{array}\right]
$$

where $\mathbf{r}_{P_{i}}$ is the position of the proximal endpoints of segment $i$ ( $\mathbf{r}_{P_{0}}$ is the position of the CoP) and $\mathbf{N}_{1}^{P_{1}}$ and $\mathbf{N}_{1}^{*}$ are respectively an interpolation matrix and a pseudo-interpolation matrix used to express the vector of generalised ground reaction [Dumas2007]. These interpolation matrices come from the use of natural coordinates $\mathbf{Q}_{i}=\left[\begin{array}{llll}\mathbf{u}_{i} & \mathbf{r}_{P_{i}} & \mathbf{r}_{D_{i}} & \mathbf{w}_{i}\end{array}\right]^{T}$ [Garcia de Jalon1994, Dumas2007] consisting, for each segment $i$, of two position vectors $\mathbf{r}_{P i}$ and $\mathbf{r}_{D_{i}}$ (i.e. the proximal $P_{i}$ and distal $D_{i}$ endpoints) and two unitary direction vectors (i.e. $\mathbf{u}_{i}$ and $\left.\mathbf{w}_{i}\right) . \mathbf{M}_{0}^{\mathbf{R}}$ can be expressed as a set of three $3 \mathrm{D}$ forces (i.e. $f_{1}^{\mathbf{M}_{0}^{\mathbf{R}}}, f_{2}^{\mathbf{M}_{0}^{\mathbf{R}}}$ and $f_{3}^{\mathbf{M}_{0}^{\mathbf{R}}}$ ) through the use of the matrix $\mathbf{B}_{1}^{*}$ [Dumas2007]: 


$$
\mathbf{M}_{0}^{\mathbf{R}}=\underbrace{\left[\begin{array}{lll}
\mathbf{w}_{1} \times \mathbf{u}_{1} & \mathbf{u}_{1} \times\left(\mathbf{r}_{P_{1}}-\mathbf{r}_{D_{1}}\right) & -\left(\mathbf{r}_{P_{1}}-\mathbf{r}_{D_{1}}\right) \times \mathbf{w}_{1}
\end{array}\right]}_{\mathbf{B}_{1}^{*}}\left[\begin{array}{c}
f_{1}^{\mathbf{M}_{0}^{\mathbf{R}}} \\
f_{2}^{\mathbf{M}_{0}^{\mathbf{R}}} \\
f_{3}^{\mathbf{M}_{0}^{\mathbf{R}}}
\end{array}\right]
$$

The vector of generalised ground reaction $\mathbf{R}$ can then be expressed as:

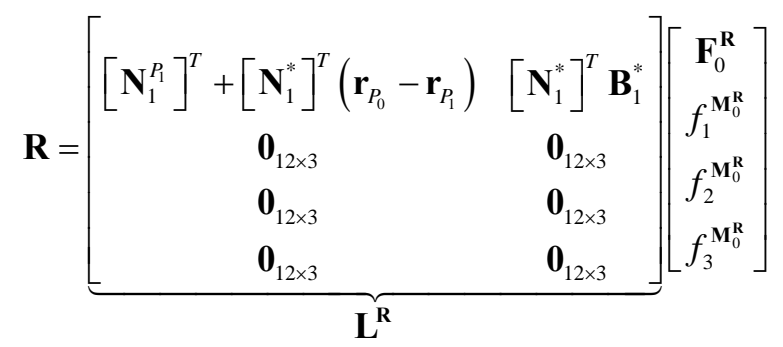

Similarly, by expressing the moment component of the dynamic wrench of segment $i$ as a set of three $3 \mathrm{D}$ forces (i.e. $f_{1}^{\mathbf{M}_{i}^{\mathbf{G}}}, f_{2}^{\mathbf{M}_{i}^{\mathbf{G}}}$ and $f_{3}^{\mathbf{M}_{i}^{\mathbf{G}}}$ ), the product of the matrix of generalised masses $\mathbf{G}$ by the vector of generalised accelerations $\ddot{\mathbf{Q}}$, as it appears in Equations 1, 2, and 3, can be expressed as:

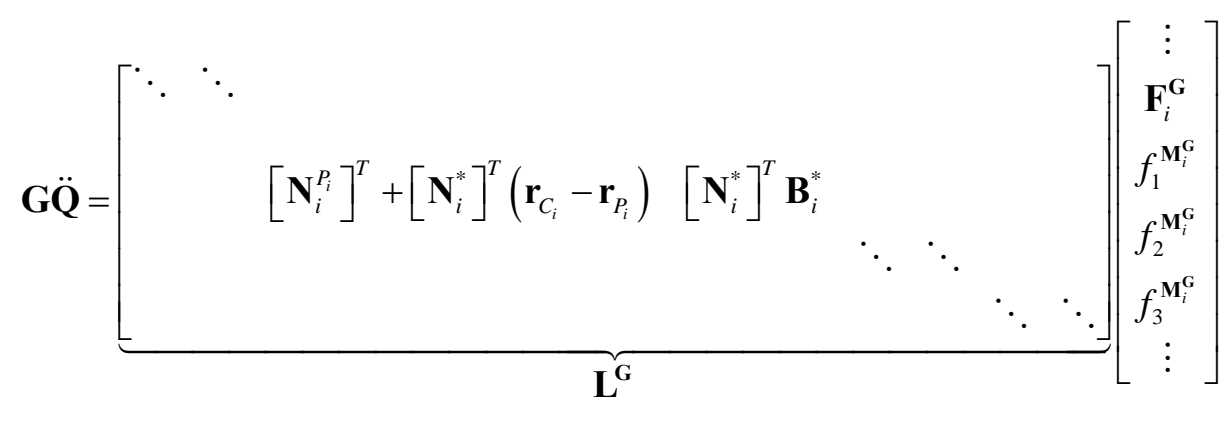

Contribution analysis

To compute individual musculo-tendon force contributions to ground reaction and dynamic wrenches, each individual musculo-tendon force is applied in isolation (i.e. $\mathbf{P}=\mathbf{0}$ and all $f^{j}=0$ except one) in Equation 2: 


$$
\left[\begin{array}{c}
\mathbf{F}_{0}^{\mathbf{R}, f^{j}} \\
f_{1}^{\mathbf{M}_{0}^{\mathbf{R}}, f^{j}} \\
f_{2}^{\mathbf{M}_{0}^{\mathbf{R}}, f^{j}} \\
f_{3}^{\mathbf{M}_{0}^{\mathbf{R}}, f^{j}} \\
\vdots \\
\mathbf{F}_{i}^{\mathbf{G}, f^{j}} \\
f_{1}^{\mathbf{M}_{i}^{\mathbf{G}}, f^{j}} \\
f_{2}^{\mathbf{M}_{i}^{\mathbf{G}}, f^{j}} \\
f_{3}^{\mathbf{M}_{i}^{\mathbf{G}}, f^{j}} \\
\vdots
\end{array}\right]=\left[\begin{array}{ll}
-\mathbf{Z}_{\mathbf{K}}{ }^{T} \mathbf{L}^{\mathbf{R}} & \mathbf{Z}_{\mathbf{K}}{ }^{T} \mathbf{L}^{\mathbf{G}}
\end{array}\right]^{\dagger} \mathbf{Z}_{\mathbf{K}}{ }^{T} \mathbf{L}\left[\begin{array}{c}
\vdots \\
0 \\
\vdots \\
f^{j} \\
\vdots \\
0 \\
\vdots
\end{array}\right]
$$

giving

$$
\mathbf{M}_{0}^{\mathbf{R}, f^{j}}=\mathbf{B}_{1}^{*}\left[\begin{array}{c}
f_{1}^{\mathbf{M}_{0}^{\mathbf{R}}, f^{j}} \\
f_{2}^{\mathbf{M}_{0}^{\mathbf{R}}, f^{j}} \\
f_{3}^{\mathbf{M}_{0}^{\mathbf{R}}, f^{j}}
\end{array}\right]
$$

and giving

$$
\ddot{\mathbf{Q}}^{f^{j}}=[\mathbf{G}]^{-1} \mathbf{L}^{G}\left[\begin{array}{c}
\vdots \\
\mathbf{F}_{i}^{\mathbf{G}, f^{j}} \\
f_{1}^{\mathbf{M}_{i}^{\mathbf{G}}, f^{j}} \\
f_{2}^{\mathbf{M}_{i}^{\mathbf{G}}, f^{j}} \\
f_{3}^{\mathbf{M}_{i}^{\mathbf{G}}, f^{j}} \\
\vdots
\end{array}\right]
$$

During the swing phase, where no ground reaction occurs, only muscles contribute to the dynamic wrench. Equation S5 is modified accordingly (while S7 remains the same):

$$
\left[\begin{array}{c}
\vdots \\
\mathbf{F}_{i}^{\mathbf{G}, f^{j}} \\
f_{1}^{\mathbf{M}_{i}^{\mathbf{G}}, f^{j}} \\
f_{2}^{\mathbf{M}_{i}^{\mathbf{G}}, f^{j}} \\
f_{3}^{\mathbf{M}_{i}^{\mathbf{G}}, f^{j}} \\
\vdots
\end{array}\right]=\left[\mathbf{Z}_{\mathbf{K}}{ }^{T} \mathbf{L}^{\mathbf{G}}\right]^{\dagger} \mathbf{Z}_{\mathbf{K}}{ }^{T} \mathbf{L}\left[\begin{array}{c}
\vdots \\
0 \\
\vdots \\
f^{j} \\
\vdots \\
\vdots \\
0
\end{array}\right]
$$

Similarly, to compute individual musculo-tendon force contributions to joint contact, ligament and bone forces (i.e. $\lambda_{1}$ ), each individual musculo-tendon force is applied in isolation (i.e. $\mathbf{P}=\mathbf{0}$ and all $f^{j}=0$ except one) in Equation 3: 


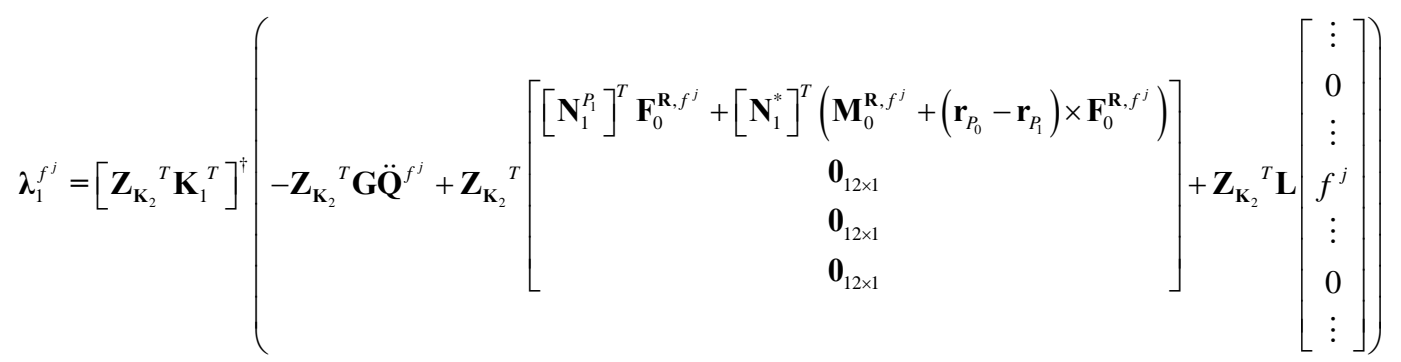

where, $\mathbf{F}_{0}^{\mathbf{R}, f^{j}}, \mathbf{M}_{0}^{\mathbf{R}, f^{j}}$ are null during the swing phase.

All 3D forces are expressed in the proximal segmental coordinate system for contacts, and along the structure longitudinal axis for bones and ligaments. 3D ground reaction force and moment are expressed in the inertial coordinate system.

\section{S3. Comprehensive results}

All results related to the present study are reported in the following figures. Both newly-determined contributions and those reported in the literature are presented. 


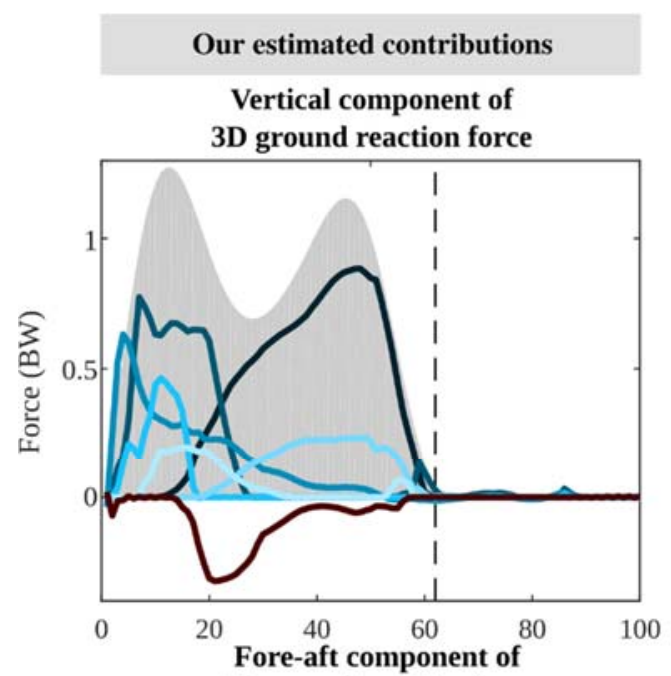

Main contributors
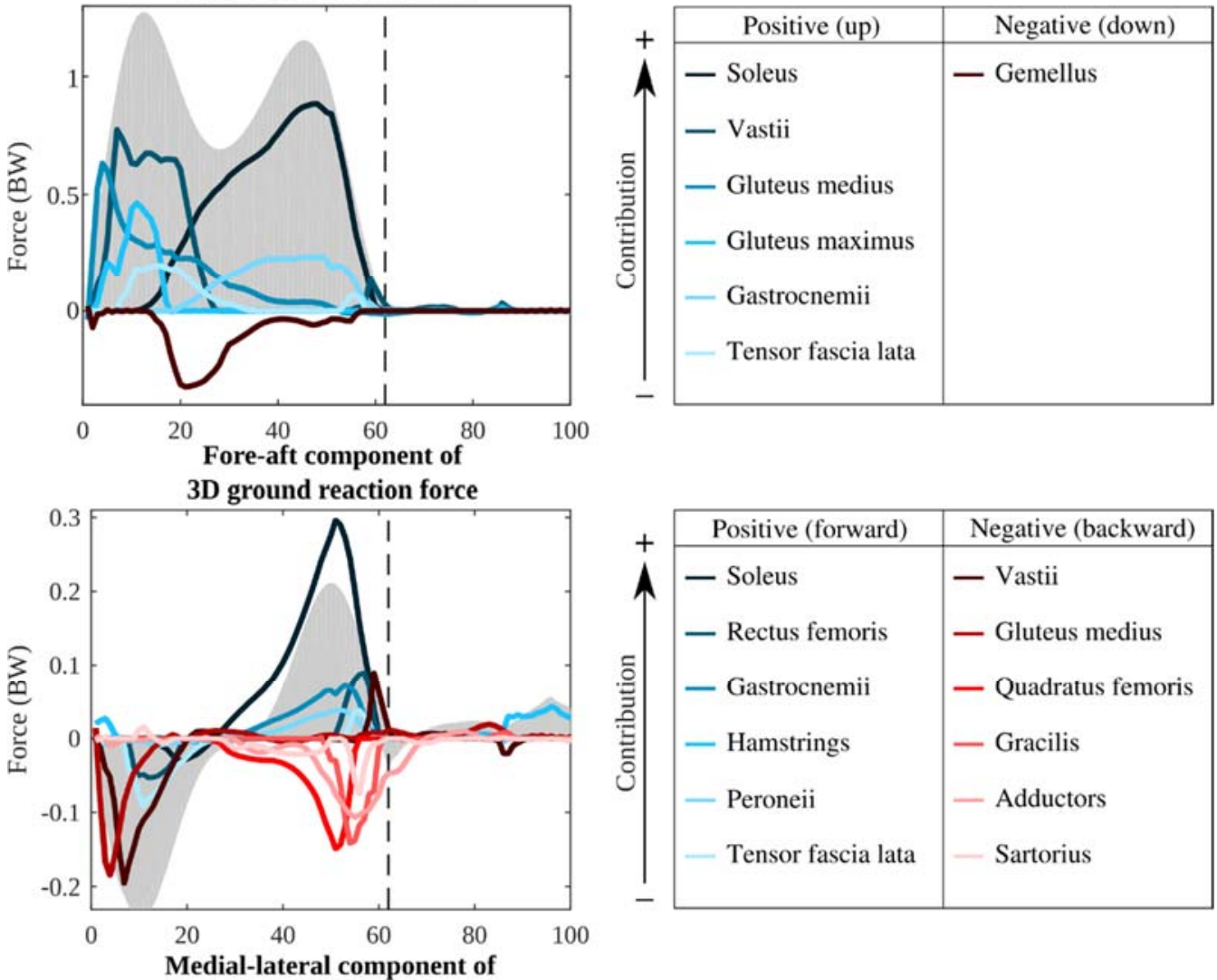

3D ground reaction force
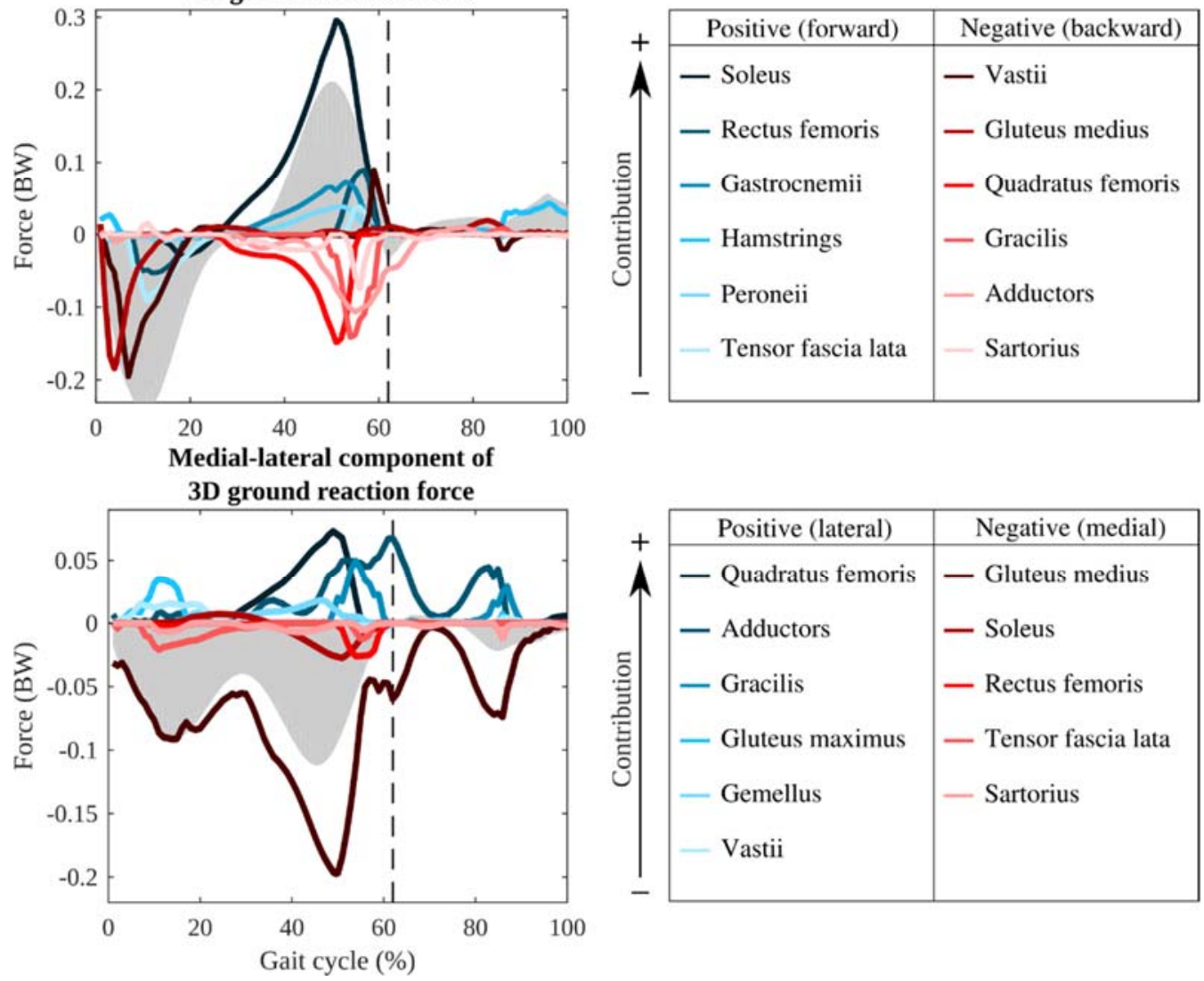

Fig. S1: Components of 3D ground reaction force (expressed in body weight BW, grey area in the figure) during a gait cycle, with the 6 major positive and negative contributions to these forces. Only muscle groups whose maximum contribution is higher than $10 \%$ of the maximum value of the investigated force are reported. These contributors are listed on the right. 


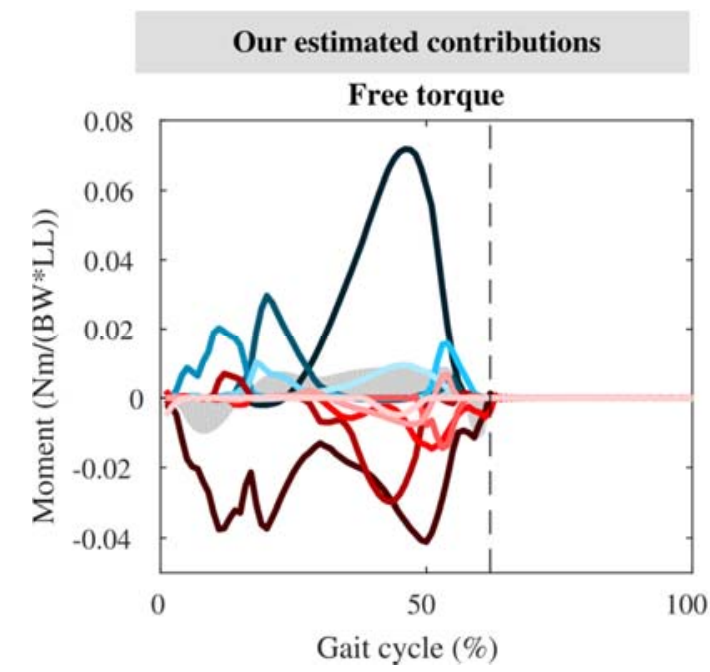

Main contributors

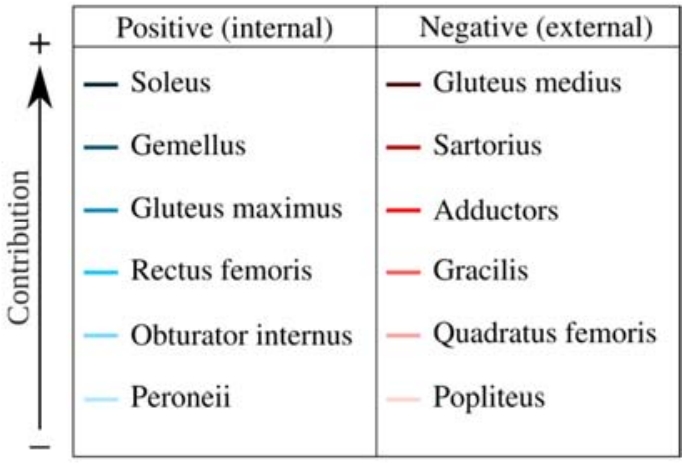

Fig. S2: Free torque (normalised in body weight BW and leg length LL, grey area in the figure) during a gait cycle, with the 6 major positive and negative contributions to this component of 3D ground reaction moment. Only muscle groups whose maximum contribution is higher than $10 \%$ of the maximum value of the investigated force are reported. These contributors are listed on the right. The grey area represents the full muscle contribution. 


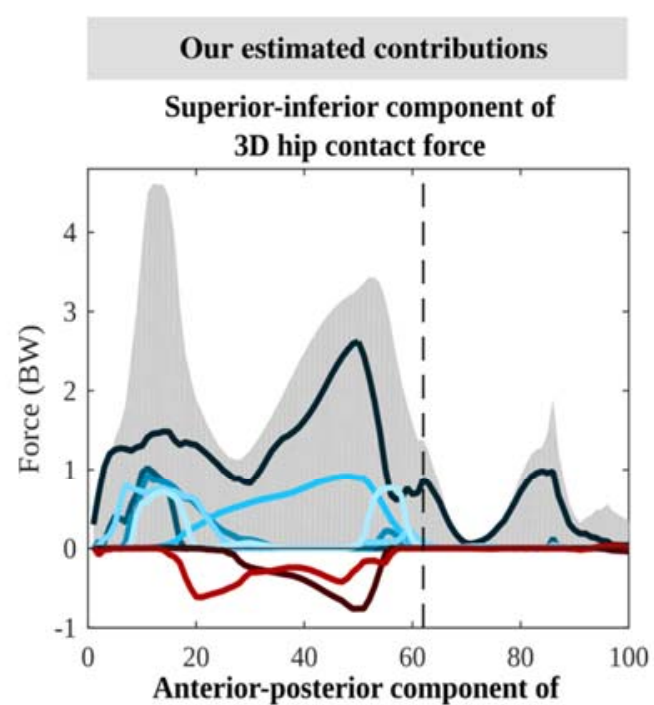

Main contributors

3D hip contact force
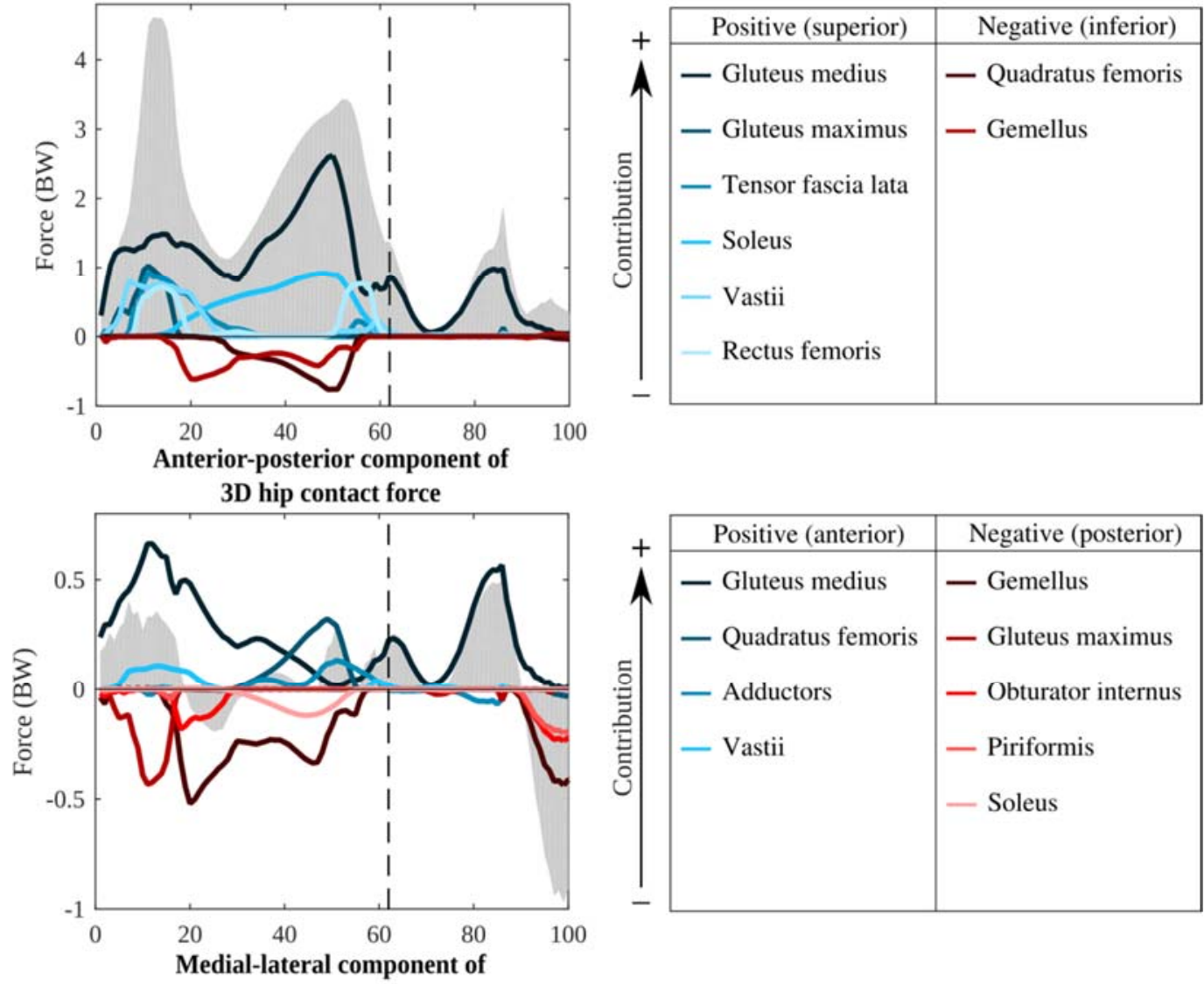

3D hip contact force
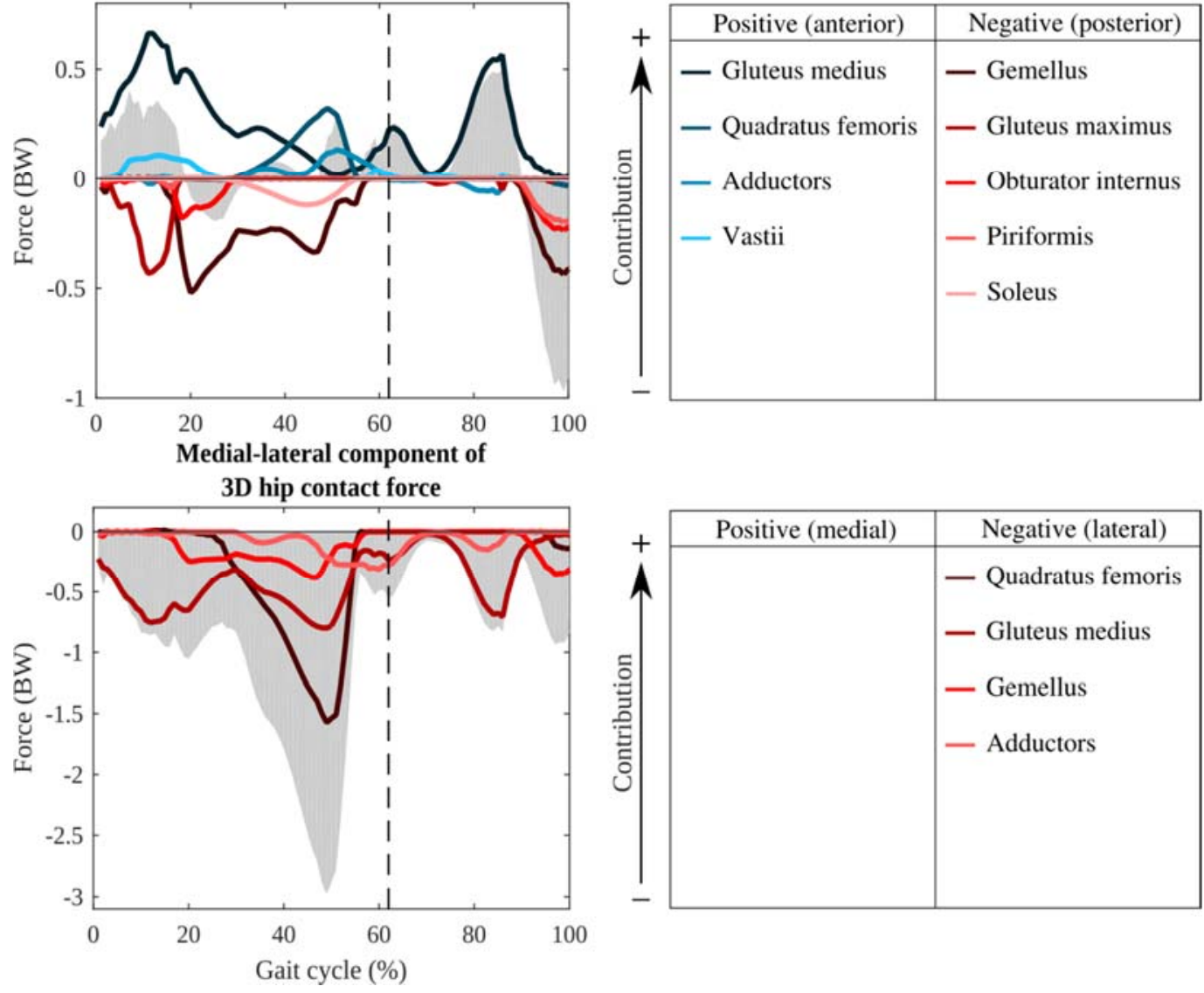

Fig. S3: Components of 3D hip contact force (expressed in body weight BW, grey area in the figure) during a gait cycle, with the 6 major positive and negative contributions to these forces. Only muscle groups whose maximum contribution is higher than $10 \%$ of the maximum value of the investigated force are reported. These contributors are listed on the right. 


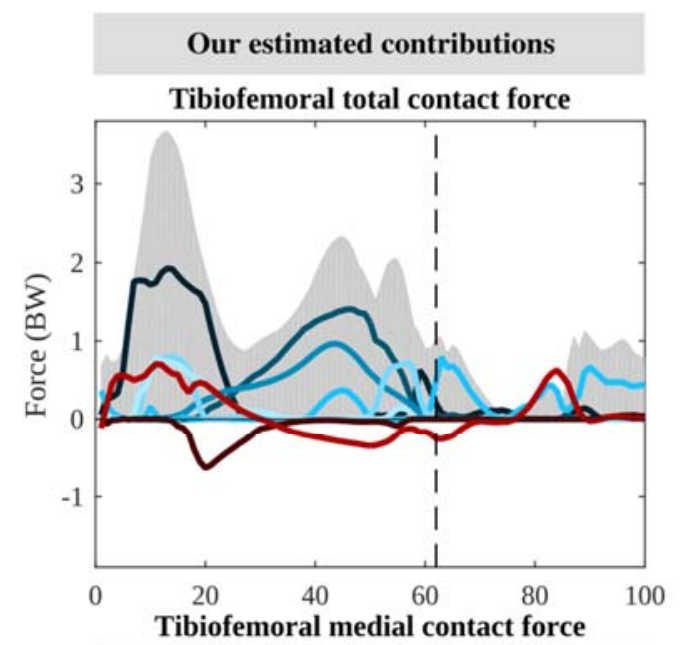

Main contributors
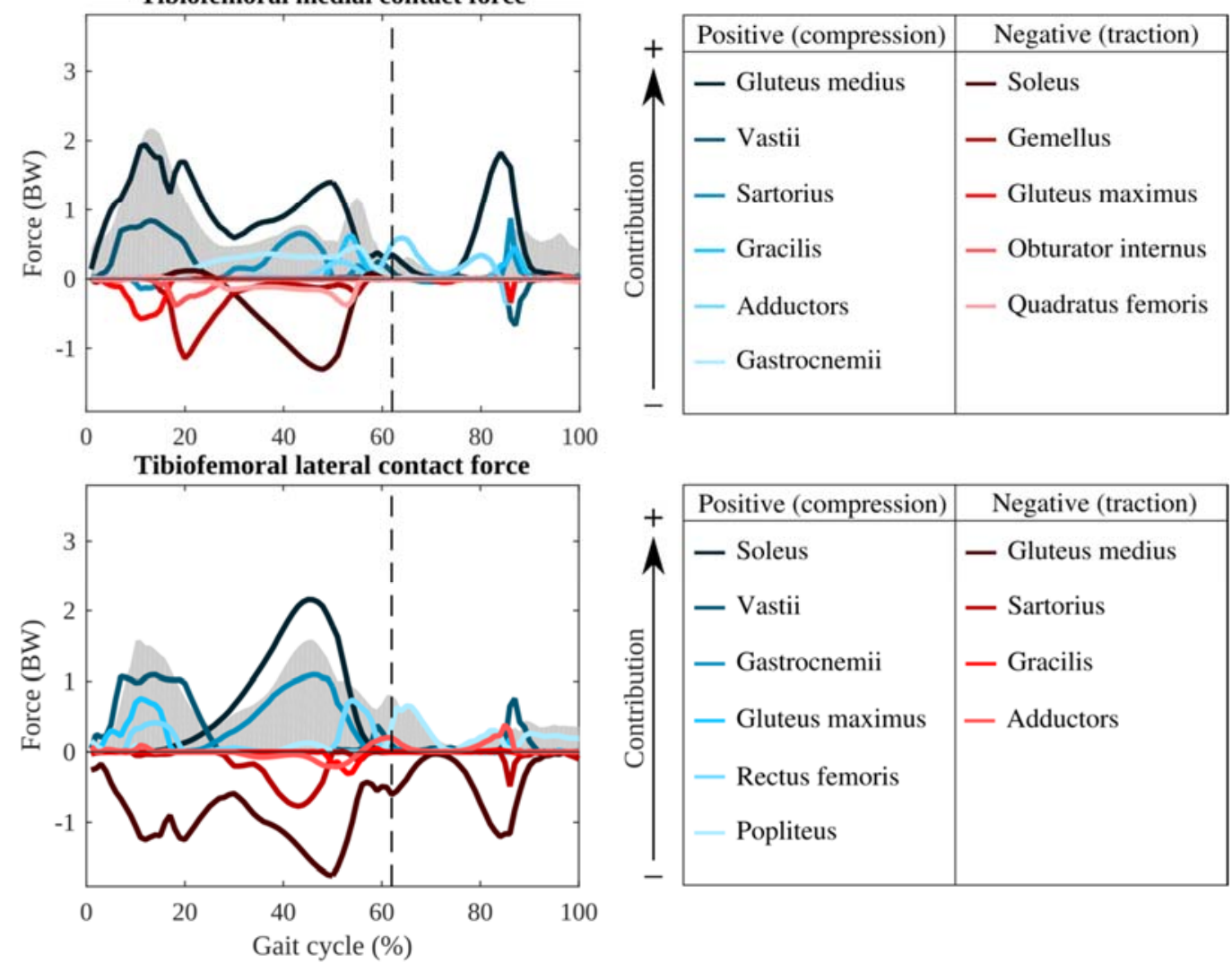

Fig. S4: Tibiofemoral total, medial and lateral contact forces (expressed in body weight BW, grey area in the figure) during a gait cycle, with the 6 major positive and negative contributions to these forces. Only muscle groups whose maximum contribution is higher than $10 \%$ of the maximum value of the investigated force are reported. These contributors are listed on the right. 


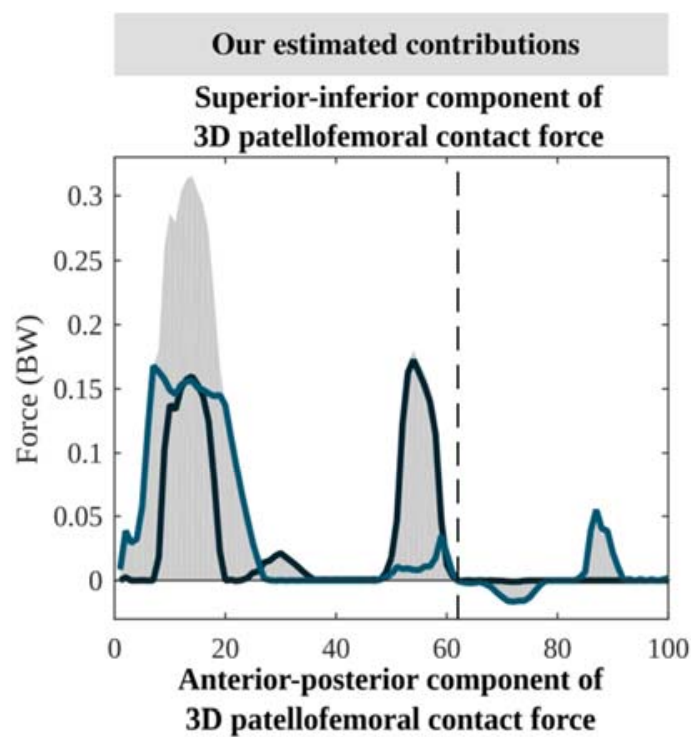

Main contributors
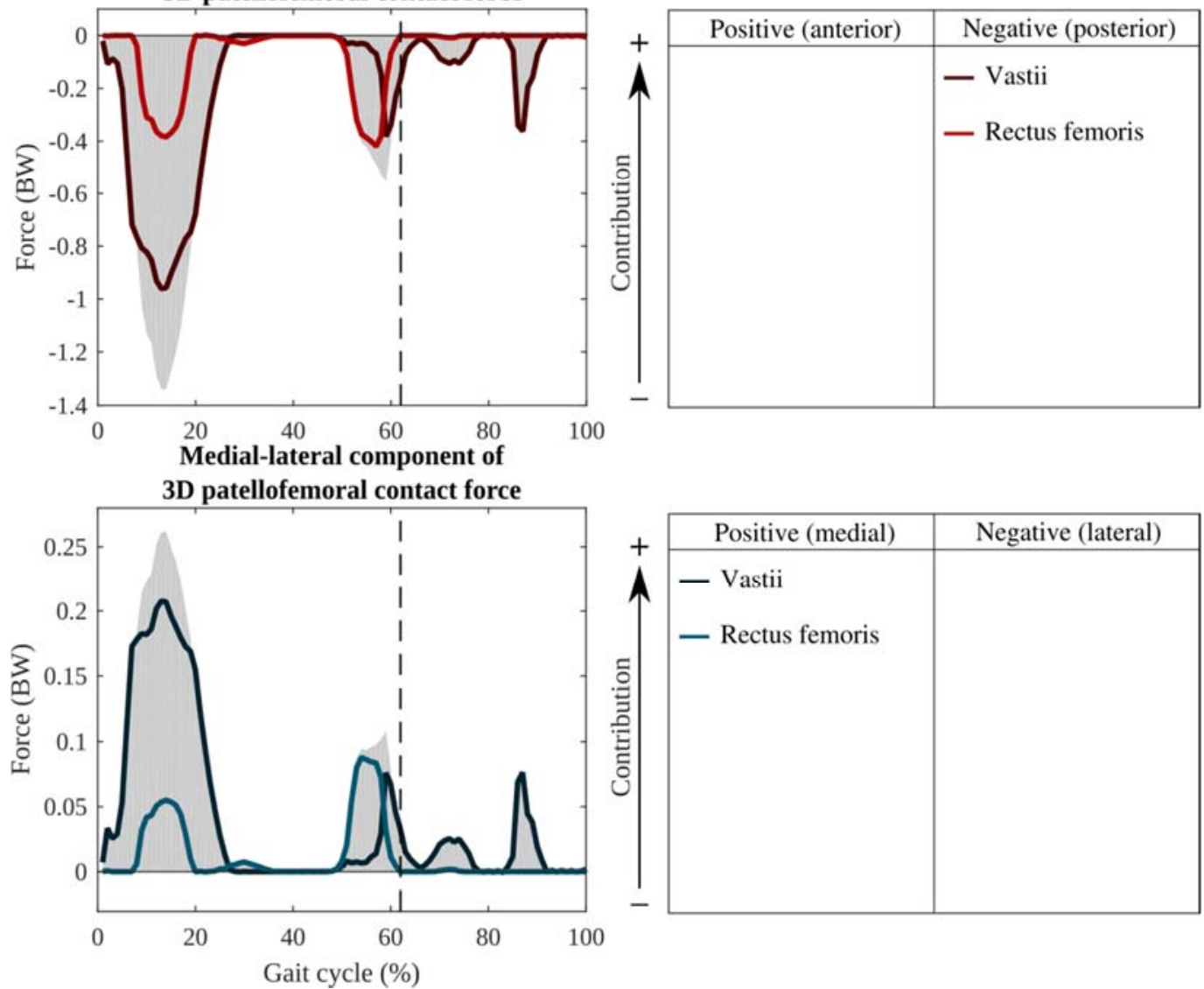

Fig. S5: Components of 3D patellofemoral contact force (expressed in body weight BW, grey area in the figure) during a gait cycle, with the 6 major positive and negative contributions to these forces. Only muscle groups whose maximum contribution is higher than $10 \%$ of the maximum value of the investigated force are reported. These contributors are listed on the right. 


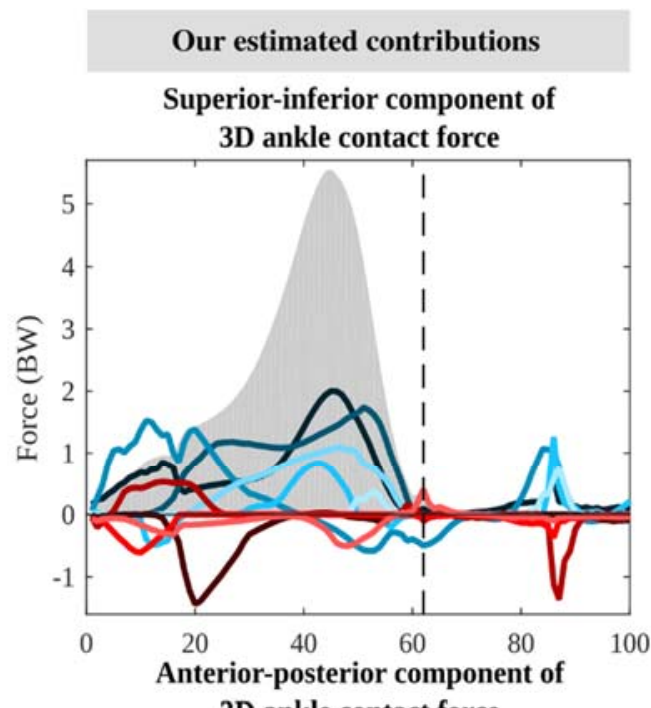

Main contributors

Superior-inferior component of

3D ankle contact force
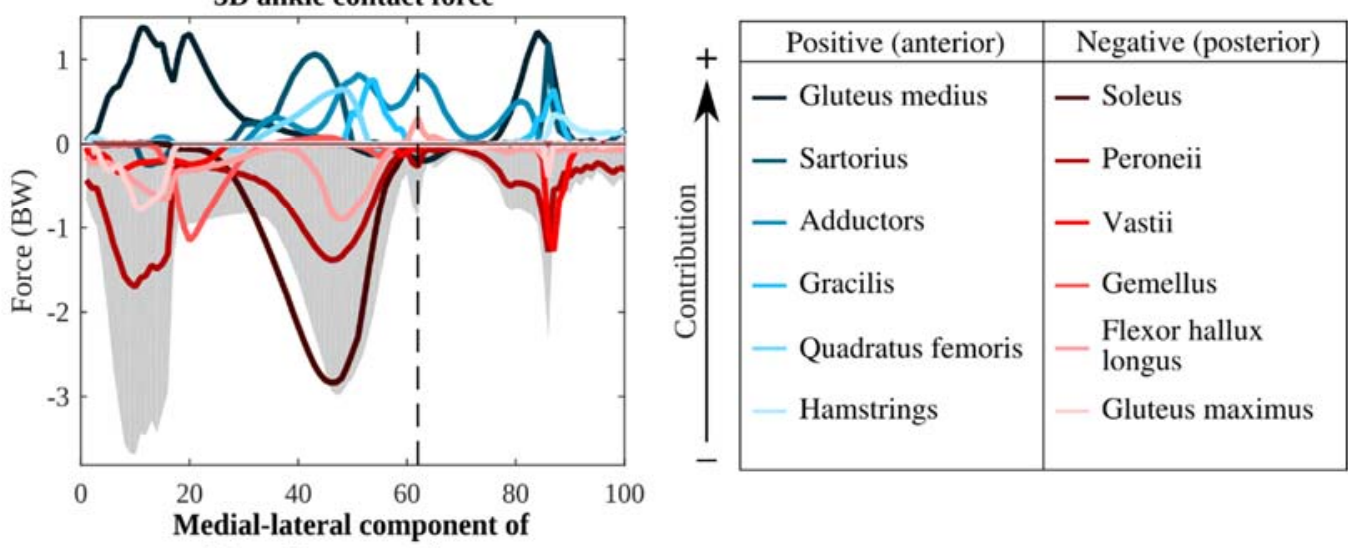

3D ankle contact force

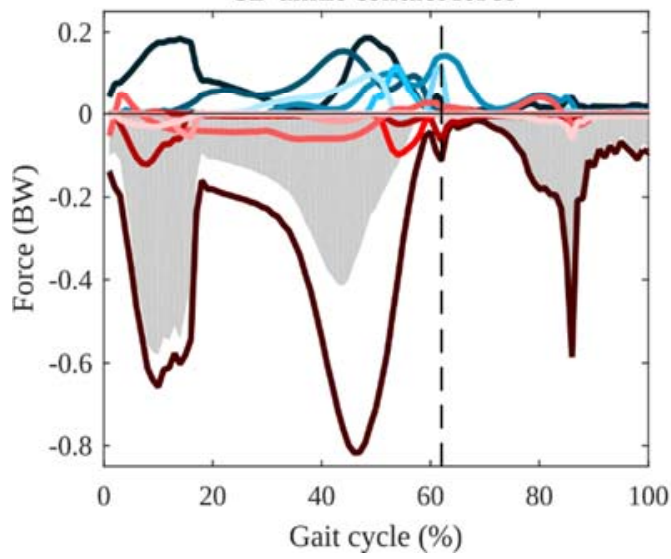

\begin{tabular}{|c|c|}
\hline Positive (superior) & Negative (inferior) \\
\hline - Peroneii & - Gemellus \\
\hline - Soleus & - Vastii \\
\hline — Gluteus medius & - Tibialis anterior \\
\hline - Sartorius & 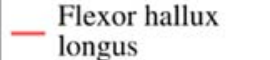 \\
\hline — Gastrocnemii & \\
\hline - Gracilis & \\
\hline
\end{tabular}

\section{0} 100

\begin{tabular}{|c|c|}
\hline Positive (medial) & Negative (lateral) \\
\hline 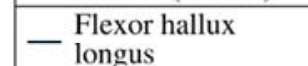 & - Peroneii \\
\hline — Soleus & $-\begin{array}{l}\text { Extensor digitorum } \\
\text { longus }\end{array}$ \\
\hline — Adductors & - Rectus femoris \\
\hline — Gracilis & — Gluteus medius \\
\hline - Plantaris & — Tibialis posterior \\
\hline — Quadratus femoris & - Tibialis anterior \\
\hline
\end{tabular}

Fig. S6: Components of 3D ankle contact force (expressed in body weight BW, grey area in the figure) during a gait cycle, with the 6 major positive and negative contributions to these forces. Only muscle groups whose maximum contribution is higher than $10 \%$ of the maximum value of the investigated force are reported. These contributors are listed on the right. 

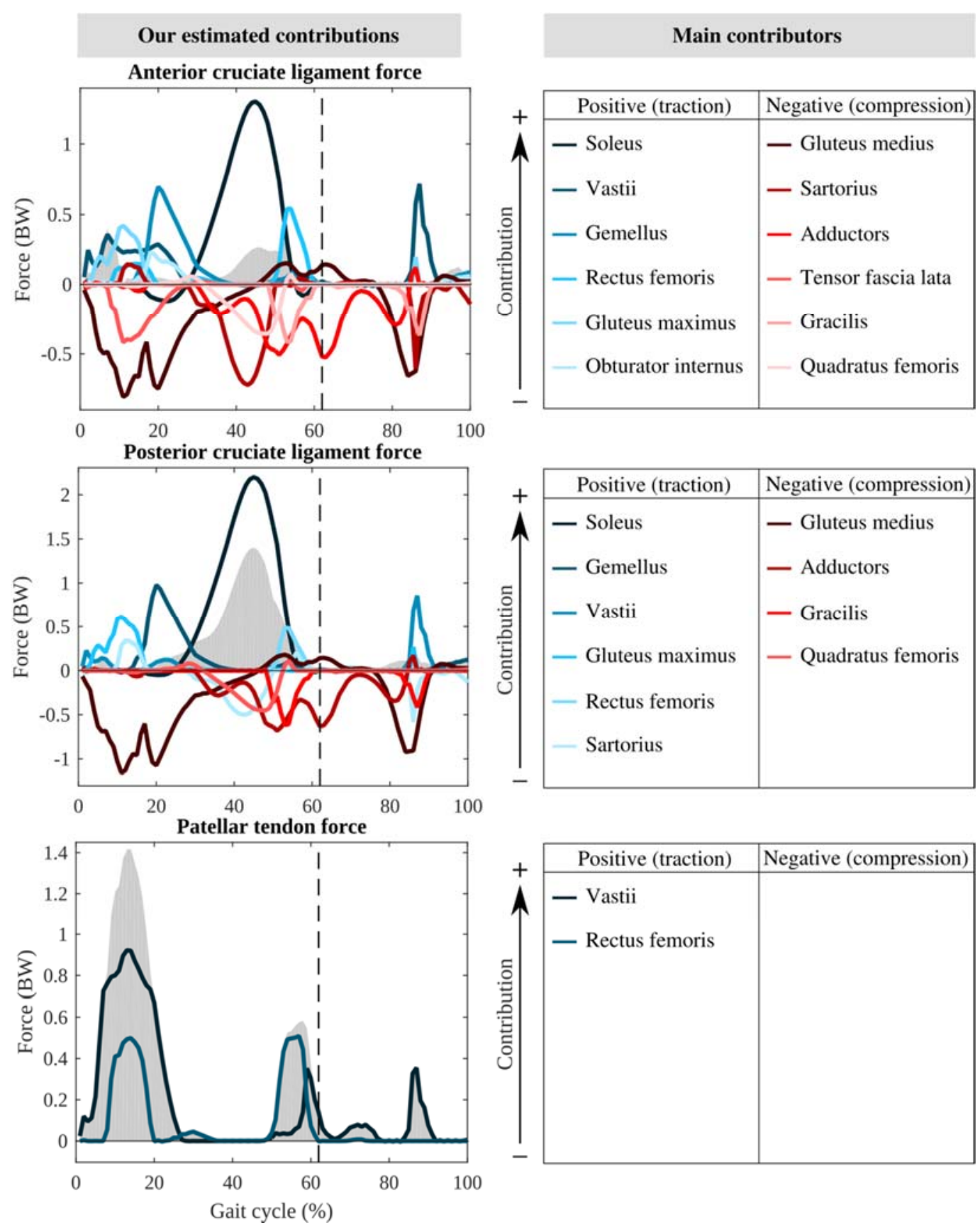

Fig. S7: Cruciate ligaments (ACL and PCL) and patellar tendon forces (expressed in body weight BW, grey area in the figure) during a gait cycle, with the 6 major positive and negative contributions to these forces. Only muscle groups whose maximum contribution is higher than $10 \%$ of the maximum value of the investigated force are reported. These contributors are listed on the right. 


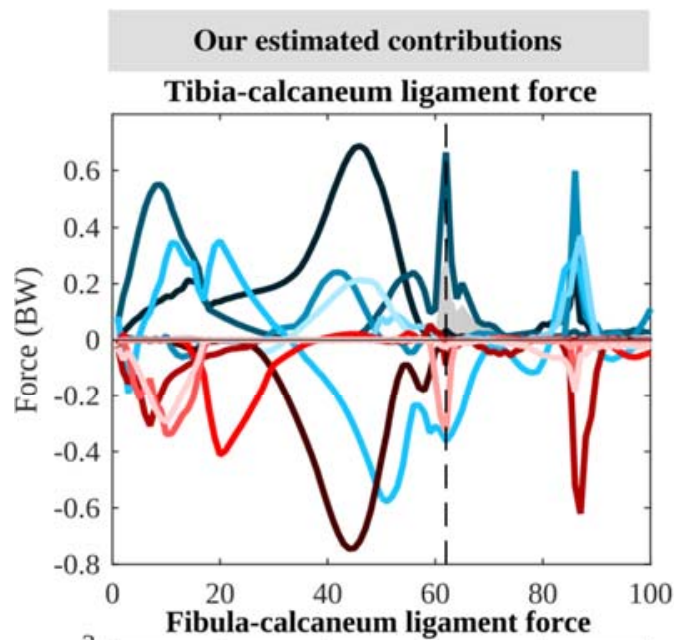

Main contributors
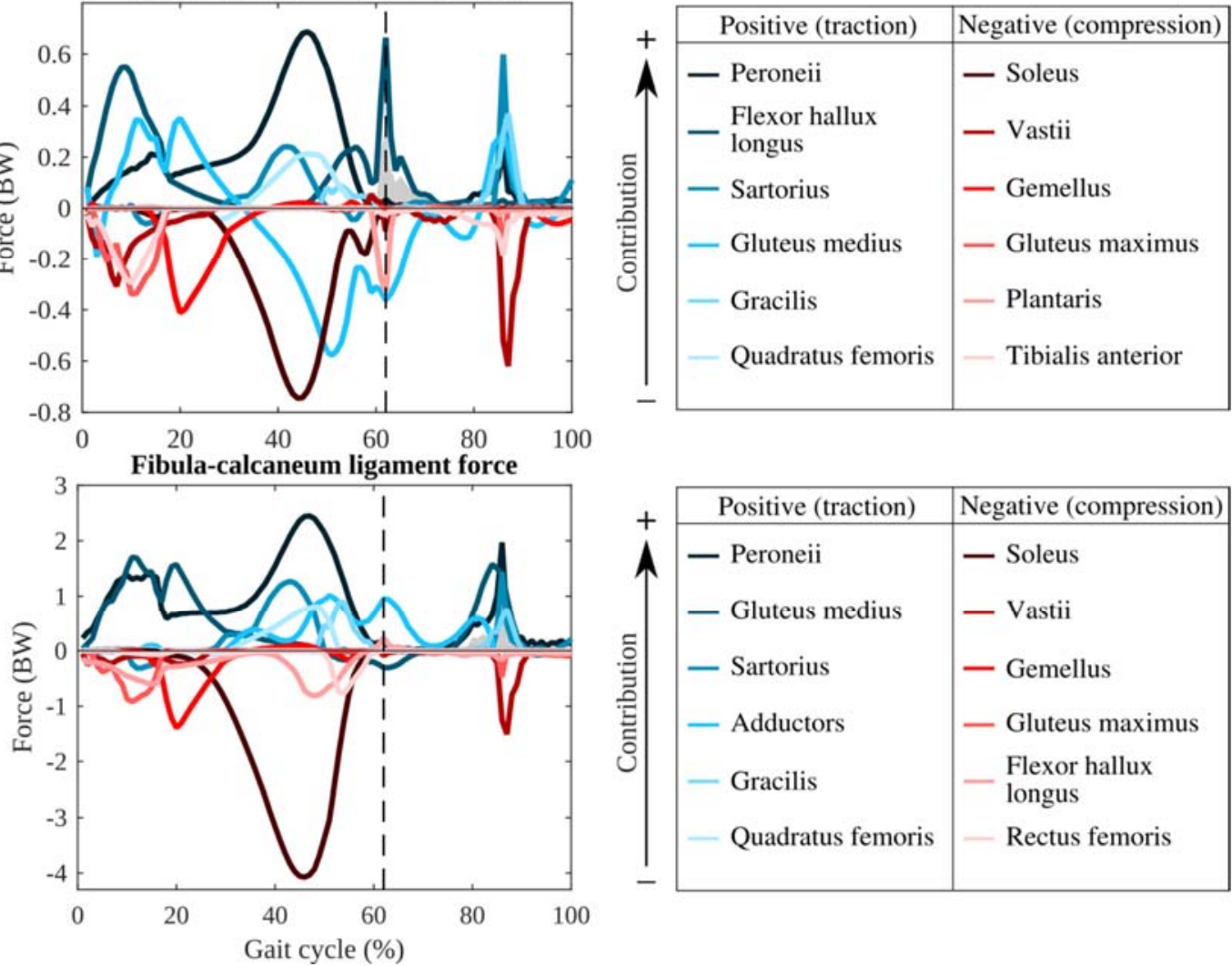

Fig. S8: Tibia-calcaneum ligament (TiCaL) and fibula-calcaneum ligament (CaFiL) ligament forces (expressed in body weight BW, grey area in the figure) during a gait cycle, with the 6 major positive and negative contributions to these forces. Only muscle groups whose maximum contribution is higher than $10 \%$ of the maximum value of the investigated force are reported. These contributors are listed on the right. 

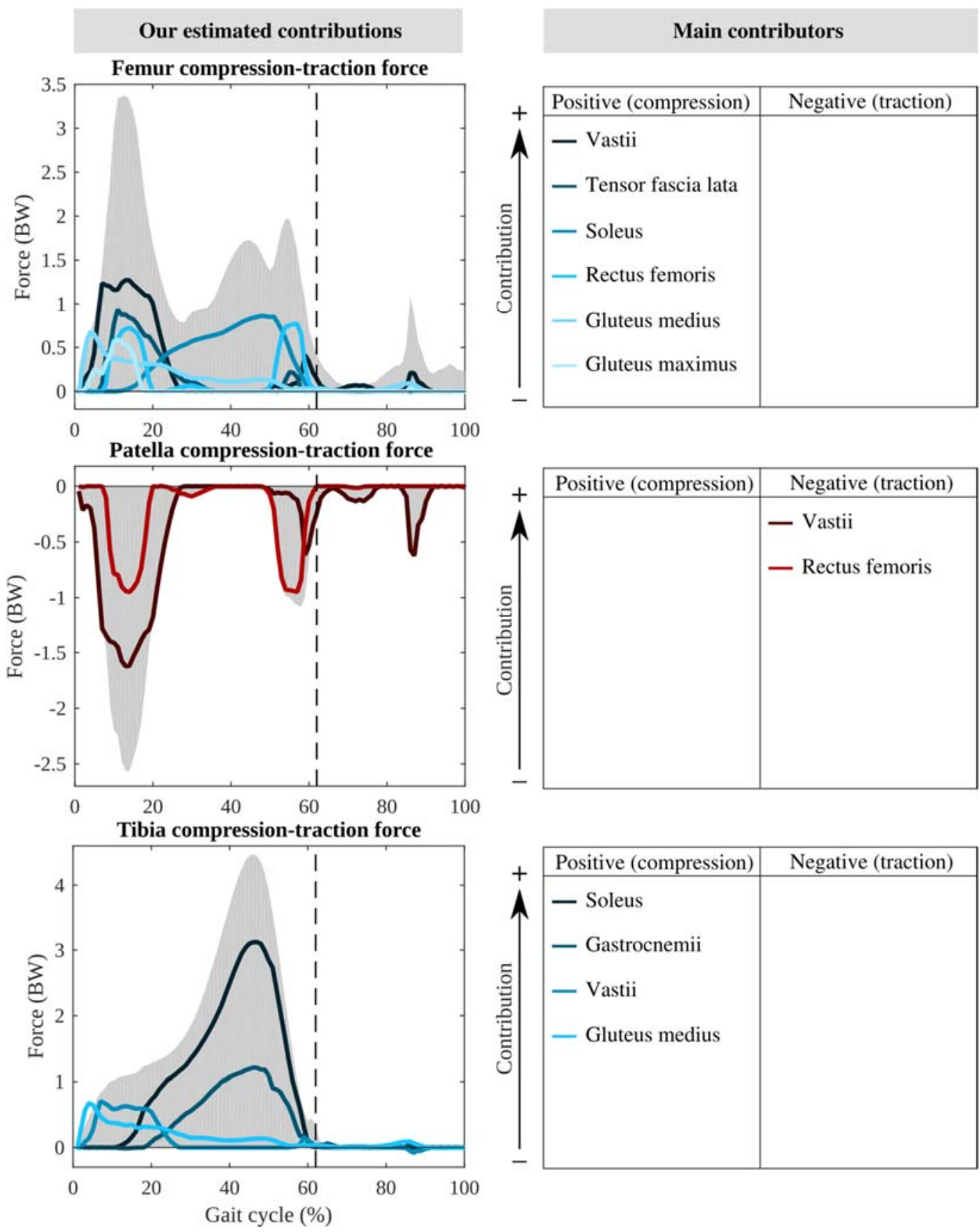

Fig. S9: Femur, patella and tibia compression-traction forces (expressed in body weight BW, grey area in the figure) during a gait cycle, with the 6 major positive and negative contributions to these forces. Only muscle groups whose maximum contribution is higher than $10 \%$ of the maximum value of the investigated force are reported. These contributors are listed on the right. 


\section{References}

[DiGregorio2007] Di Gregorio, R., V. Parenti-Castelli, J. J. O’Connor, and A. Leardini.

Mathematical models of passive motion at the human ankle joint by equivalent spatial parallel mechanisms. Med. Biol. Eng. Comput. 45:305-13, 2007.

[Dumas2007] Dumas, R., and L. Chèze. 3D inverse dynamics in non-orthonormal segment coordinate system. Med. Biol. Eng. Comput. 45:315-22, 2007.

[Dumas2012] Dumas, R., F. Moissenet, X. Gasparutto, and L. Chèze. Influence of joint models on lower-limb musculo-tendon forces and three-dimensional joint reaction forces during gait. Proc. Inst. Mech. Eng. H. 226:146-60, 2012.

[Feikes2003] Feikes, J. D., J. J. O’Connor, and A. B. Zavatsky. A constraint-based approach to modelling the mobility of the human knee joint. J. Biomech. 36:125-9, 2003.

[Garcia de Jalon1994] Garcia de Jalon, J., and E. Bayo. Kinematic and dynamic simulation of multibody systems. The real-time challenge. Springer-Verlag, New-York, 1994.

[Lin2011] Lin, Y. C., H. J. Kim, and M. G. Pandy. A computationally efficient method for assessing muscle function during human locomotion. Int. J. Numer. Methos Biomed. Eng. 27:436449, 2011.

[Moissenet2012] Moissenet, F., L. Chèze, and R. Dumas. Anatomical kinematic constraints: consequences on musculo-tendon forces and joint reactions. Multibody Syst. Dyn. 28:125-141, 2012.

[Sancisi2011] Sancisi, N., and V. Parenti-Castelli. A New Kinematic Model of the Passive Motion of the Knee Inclusive of the Patella. J. Mech. Robot. 3:041003, 2011. 